\title{
Asymmetric Addition of Alkylzinc Reagents to Cyclic $\alpha, \beta$-Unsaturated Ketones and a Tandem Enantioselective Addition/Diastereoselective Epoxidation with Dioxygen
}

\author{
Sang-Jin Jeon and Patrick J. Walsh* \\ P. Roy and Diane T. Vagelos Laboratories, \\ University of Pennsylvania, Department of Chemistry \\ 231 South $34^{\text {th }}$ Street, Philadelphia, PA 19104-6323.
}

\section{Supporting Information}

$\begin{array}{ll}\text { Chart with the Structures and Compound Labels } & \text { S2 }\end{array}$

$\begin{array}{ll}\text { General Methods } & \text { S3 }\end{array}$

$\begin{array}{ll}\text { Synthesis of Substrate Ketones } & \text { S3 }\end{array}$

Enantioselective Addition of Dialkylzinc Reagents to Cyclic Enones S3

$\begin{array}{ll}\text { Characterization of S1-S12 } & \text { S4 }\end{array}$

$\begin{array}{ll}\text { One-pot Synthesis of Epoxy Alcohols } & \text { S7 }\end{array}$

$\begin{array}{ll}\text { Characterization of S13-S19 } & \text { S8 }\end{array}$

Semipinacol Rearrangement Reaction of Epoxy Alcohols with $\mathrm{BF}_{3} \cdot \mathrm{OEt}_{2} \quad \mathrm{~S} 11$

$\begin{array}{ll}\text { Characterization of S20-S23 } & \text { S11 }\end{array}$

Conditions for the Determination of Enantiomeric Excesses $\quad \mathrm{S} 12$

$\begin{array}{ll}\text { References } & \text { S15 }\end{array}$

${ }^{1} \mathrm{H}$ and ${ }^{13} \mathrm{C}\left\{{ }^{1} \mathrm{H}\right\}$ NMR spectra (S1-S23) $\quad$ S16 


\section{Chart with the Structures and Compound Labels}

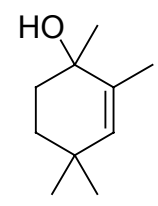

S1

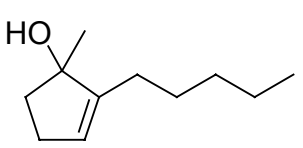

S5<smiles>CC1(O)CCCC/C1=C\c1ccccc1</smiles>

S9<smiles>CC1(O)CCC(C)(C)C2(C)OC12</smiles>

S13<smiles>CCCCCC12OC1CCC2(C)O</smiles>

S17<smiles>CCCCCC1(CC)C(=O)CCC1O</smiles>

S21

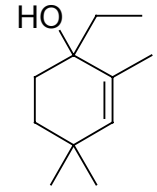

S2<smiles>CCCCCC1=CCCC1(O)CC</smiles>

S6<smiles>CCC1(O)CCCC/C1=C\c1ccccc1</smiles>

S10

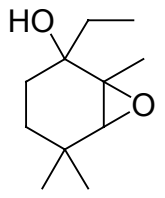

S14

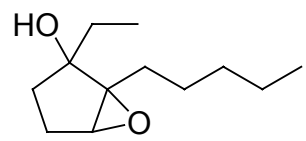

S18<smiles>CC1(C)C(=O)CCC1O</smiles>

S22

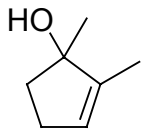

S3<smiles>CC1(O)CCCC=C1c1ccccc1</smiles>

S7<smiles>CCC1(O)C=CCCC1</smiles>

S11

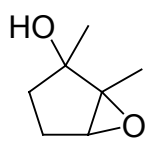

S15<smiles></smiles>

S19<smiles>CCC1(C)C(=O)CCC1O</smiles>

S23

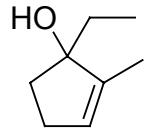

S4<smiles>OC1(Cc2ccccc2)CCCC=C1c1ccccc1</smiles>

S8<smiles>CCC1(O)C=C(C)CC(C)(C)C1</smiles>

S12

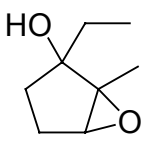

S16

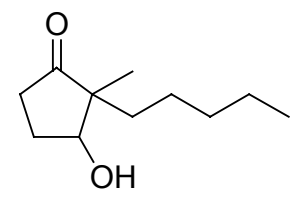

S20 
General Methods. All reactions using dialkylzinc and titanium(IV) isopropoxide were carried out in a Vacuum Atmospheres dry box or under nitrogen using standard Schlenk techniques. NMR spectra were obtained on a Brüker $500 \mathrm{MHz}$ Fourier transform spectrometer at the University of Pennsylvania NMR facility. ${ }^{1} \mathrm{H}$ NMR spectra were referenced to tetramethylsilane in $\mathrm{CDCl}_{3} ;{ }^{13} \mathrm{C}\left\{{ }^{1} \mathrm{H}\right\}$ NMR spectra were referenced to residual solvent. All reagents were obtained from Aldrich Chemical Company; ketone substrates were obtained from Aldrich or Acros Organics unless otherwise specified. Titanium(IV) isopropoxide and all liquid ketone substrates were distilled prior to use. 1.0 $\mathrm{M}$ dialkylzinc and 1.4 M titanium(IV) isopropoxide solutions were prepared and stored in a Vacuum Atmospheres dry box.

\section{Synthesis of Substrate Ketones.}

Preparation of 2-Phenylcyclohexen-1-one. 2-Phenylcyclohexen-1-one was prepared according to literature procedure. ${ }^{1}$

Preparation of 2-Benzylidenecyclohexanone. 2-Benzylidenecyclohexanone was prepared according to literature procedure. ${ }^{2}$

\section{Enantioselective Addition of Dialkylzinc Reagents to Cyclic Enones.}

General Procedure A. The bis(sulfonamide) ligand (10 mol\%) was weighed into the reaction vessel, and the dialkylzinc (1.0 $\mathrm{M}$ toluene solution, 3 equiv) and the titanium(IV) isopropoxide (1.4 M hexane solution, 1.2 equiv) were added at room temperature. After 5 min, the substrate ketone (1.0 equiv) was added neat. The homogeneous reaction mixture was stirred at room temperature. After completion, it was quenched with small amount of water (1-1.5 mL), dried over $\mathrm{MgSO}_{4}$, concentrated under reduced pressure, and purified by column chromatography on silica gel. 
Preparation of 1,2,4,4-Tetramethyl-cyclohex-2-enol (S1). General procedure A was applied to 2,4,4-trimethyl-2-cyclohexen-1-one on a $77 \mu \mathrm{L}(0.5 \mathrm{mmol})$ scale, using 1.5 $\mathrm{mL}$ of dimethylzinc solution. The crude product was purified by column chromatography on silica gel (hexanes / EtOAc : 95 / 5) to give S1 (65 mg, 84\% yield, 99\% ee) as an oil: $[\alpha]_{\mathrm{D}}{ }^{20}=+37.5\left(c 1.20, \mathrm{CHCl}_{3}\right) ;{ }^{1} \mathrm{H}$ NMR $\left(\mathrm{C}_{6} \mathrm{D}_{6}, 500 \mathrm{MHz}\right) \delta 0.87(\mathrm{~s}, 3 \mathrm{H}), 0.92(\mathrm{~s}, 3 \mathrm{H})$, 1.17 (s, 3H), 1.32 (ddd, $J=12.9,8.7,3.8 \mathrm{~Hz}, 1 \mathrm{H}), 1.44$ (ddd, $J=12.9,8.7,3.8 \mathrm{~Hz}, 1 \mathrm{H})$, 1.58-1.67 (m, 2H), $1.68(\mathrm{~s}, 3 \mathrm{H}), 5.04(\mathrm{~s}, 1 \mathrm{H}) \mathrm{ppm} ;{ }^{13} \mathrm{C}\left\{{ }^{1} \mathrm{H}\right\} \mathrm{NMR}\left(\mathrm{C}_{6} \mathrm{D}_{6}, 125 \mathrm{MHz}\right) \delta 17.9$, 27.1, 29.3, 29.9, 32.4, 34.7, 37.0, 69.9, 134.7, 136.7 ppm; IR (NaCl) 3363, 2958, 2859, 1717, 1454, 1360, $1067 \mathrm{~cm}^{-1}$; HRMS calcd for $\mathrm{C}_{10} \mathrm{H}_{16}\left(\mathrm{M}-\mathrm{H}_{2} \mathrm{O}\right)^{+}$: 136.1252, found 136.1258.

Preparation of 1-Ethyl-2,4,4-trimethyl-cyclohex-2-enol (S2). General procedure A was applied to 2,4,4-trimethyl-2-cyclohexen-1-one on a $77 \mu \mathrm{L}(0.5 \mathrm{mmol})$ scale, using $1.5 \mathrm{~mL}$ of diethylzinc solution. The crude product was purified by column chromatography on silica gel (hexanes / EtOAc : 95 / 5) to give S2 (64 mg, 76\% yield, 98\% ee) as an oil: $[\alpha]_{\mathrm{D}}{ }^{20}=+65.5\left(c 1.13, \mathrm{CHCl}_{3}\right) ;{ }^{1} \mathrm{H} \mathrm{NMR}\left(\mathrm{C}_{6} \mathrm{D}_{6}, 500 \mathrm{MHz}\right) \delta 0.80(\mathrm{t}, J=7.5 \mathrm{~Hz}, 3 \mathrm{H}), 0.88$ $(\mathrm{s}, 3 \mathrm{H}), 0.93(\mathrm{~s}, 3 \mathrm{H}), 1.27-1.32(\mathrm{~m}, 1 \mathrm{H}), 1.42-1.60(\mathrm{~m}, 4 \mathrm{H}), 1.65(\mathrm{~s}, 3 \mathrm{H}), 1.75(\mathrm{ddd}, J=$ 14.2, 11.2, 3.1 Hz, 1H), $5.14(\mathrm{~s}, 1 \mathrm{H}) \mathrm{ppm} ;{ }^{13} \mathrm{C}\left\{{ }^{1} \mathrm{H}\right\} \mathrm{NMR}\left(\mathrm{C}_{6} \mathrm{D}_{6}, 125 \mathrm{MHz}\right) \delta 8.5,17.9$, 28.4, 30.6, 31.7, 31.9, 32.2, 33.9, 72.0, 135.9, 136.3 ppm; IR (NaCl) 3394, 2953, 1716, 1455, 1360, $1234 \mathrm{~cm}^{-1}$; HRMS calcd for $\mathrm{C}_{11} \mathrm{H}_{18}\left(\mathrm{M}-\mathrm{H}_{2} \mathrm{O}\right)^{+}:$150.1408, found 150.1361.

Preparation of 1,2-Dimethyl-cyclopent-2-enol (S3). ${ }^{3}$ General procedure A was applied to 2-methyl-2-cyclopenten-1-one on a $150 \mu \mathrm{L}(1.5 \mathrm{mmol}) \mathrm{scale}$, using $4.5 \mathrm{~mL}$ of dimethylzinc solution. The crude product was purified by column chromatography on silica gel (hexanes / EtOAc : $93 / 7$ ) to give $\mathbf{S 3}\left(92.5 \mathrm{mg}, 55 \%\right.$ yield, $98 \%$ ee) as an oil: $[\alpha]_{\mathrm{D}}{ }^{20}=$ $+9.08\left(c\right.$ 1.30, $\left.\mathrm{CHCl}_{3}\right)$. Spectral data for this compound is consistent with the previous charactarization. $^{3}$ 
Preparation of 1-Ethyl-2-methyl-cyclopent-2-enol (S4). General procedure A was applied to 2-methyl-2-cyclopenten-1-one on a $100 \mu \mathrm{L}(1.0 \mathrm{mmol})$ scale, using $3 \mathrm{~mL}$ of diethylzinc solution. The crude product was purified by column chromatography on silica gel (hexanes / EtOAc : $93 / 7)$ to give $\mathbf{S 4}\left(82 \mathrm{mg}, 65 \%\right.$ yield, $96 \%$ ee) as an oil: $[\alpha]_{\mathrm{D}}{ }^{20}=$ $+15.7\left(c\right.$ 1.0, $\left.\mathrm{CHCl}_{3}\right) ;{ }^{1} \mathrm{H} \mathrm{NMR}\left(\mathrm{C}_{6} \mathrm{D}_{6}, 500 \mathrm{MHz}\right) \delta 0.81(\mathrm{t}, J=7.5 \mathrm{~Hz}, 3 \mathrm{H}), 1.39-1.46(\mathrm{~m}$, $1 \mathrm{H}), 1.58(\mathrm{~s}, 3 \mathrm{H}), 1.58-1.71(\mathrm{~m}, 2 \mathrm{H}), 1.92-1.99(\mathrm{~m}, 2 \mathrm{H}), 2.10-2.16(\mathrm{~m}, 1 \mathrm{H}), 5.29(\mathrm{~s}, 1 \mathrm{H})$ ppm; ${ }^{13} \mathrm{C}\left\{{ }^{1} \mathrm{H}\right\}$ NMR $\left(\mathrm{C}_{6} \mathrm{D}_{6}, 125 \mathrm{MHz}\right) \delta 8.7,11.6,29.1,31.3,37.4,86.2,126.9,144.0 \mathrm{ppm}$; IR (NaCl) 3371, 3036, 2963, 2927, 2854, 1699, 1461, $1377 \mathrm{~cm}^{-1}$; HRMS calcd for $\mathrm{C}_{8} \mathrm{H}_{13}$ $(\mathrm{M}-\mathrm{OH})^{+}:$109.1017, found 109.1022 .

Preparation of 1-Methyl-2-pentyl-cyclopent-2-enol (S5). ${ }^{4}$ General procedure A was applied to 2-pentyl-2-cyclopenten-1-one on a $83 \mu \mathrm{L}(0.50 \mathrm{mmol})$ scale, using $1.5 \mathrm{~mL}$ of dimethylzinc solution. Chromatography on silica gel (hexanes / EtOAc : 93 / 7) to give S5 (52.2 mg, 62\% yield, 99\% ee) as an oil: $[\alpha]_{\mathrm{D}}{ }^{20}=+7.57\left(c\right.$ 0.77, $\left.\mathrm{CHCl}_{3}\right)$. Spectral data for this compound is consistent with the previous charactarization. ${ }^{4}$

Preparation of 1-Ethyl-2-pentyl-cyclopent-2-enol (S6). General procedure A was applied to 2-pentyl-2-cyclopenten-1-one on a $83 \mu \mathrm{L}(0.50 \mathrm{mmol})$ scale, using $1.5 \mathrm{~mL}$ of diethylzinc solution. The crude product was purified by column chromatography on silica gel (hexanes / EtOAc : $93 / 7)$ to give $\mathbf{S 6}\left(45.6 \mathrm{mg}, 50 \%\right.$ yield, $99.0 \%$ ee) as an oil: $[\alpha]_{\mathrm{D}}{ }^{20}=$ $+10.8\left(c\right.$ 0.67, $\left.\mathrm{CHCl}_{3}\right) ;{ }^{1} \mathrm{H} \mathrm{NMR}\left(\mathrm{C}_{6} \mathrm{D}_{6}, 500 \mathrm{MHz}\right) \delta 0.84(\mathrm{t}, J=7.5 \mathrm{~Hz}, 3 \mathrm{H}), 0.91(\mathrm{t}, J=$ $6.9 \mathrm{~Hz}, 3 \mathrm{H}), 1.27-1.33(\mathrm{~m}, 4 \mathrm{H}), 1.44-1.54(\mathrm{~m}, 3 \mathrm{H}), 1.63-1.73(\mathrm{~m}, 2 \mathrm{H}), 1.85-2.08$ (m, 4H), 2.13-2.22 (m, 1H), $5.35(\mathrm{~m}, 1 \mathrm{H}) \mathrm{ppm} ;{ }^{13} \mathrm{C}\left\{{ }^{1} \mathrm{H}\right\}$ NMR $\left(\mathrm{C}_{6} \mathrm{D}_{6}, 125 \mathrm{MHz}\right) \delta 8.7,14.3,23.0$, 26.1, 28.2, 29.2, 31.5, 32.4, 37.7, 86.6, 125.2, 148.6 ppm; IR ( NaCl) 3370, 3059, 2925, 2856, 1699, 1461, 1073, $1033 \mathrm{~cm}^{-1}$; HRMS calcd for $\mathrm{C}_{12} \mathrm{H}_{20}\left(\mathrm{M}-\mathrm{H}_{2} \mathrm{O}\right)^{+}: 164.1565$, found 164.1557.

Preparation of 1-Methyl-2-phenyl-cyclohex-2-enol (S7). General procedure A was applied to 2-phenylcyclohexen-1-one ${ }^{1}$ on a $86.2 \mathrm{mg}(0.5 \mathrm{mmol})$ scale, using $1.5 \mathrm{~mL}$ of 
dimethylzinc solution. The crude product was purified by column chromatography on silica gel (hexanes / EtOAc : $95 / 5)$ to give $\mathbf{S 7}\left(51 \mathrm{mg}, 54 \%\right.$ yield, $95 \%$ ee) as an oil: $[\alpha]_{\mathrm{D}}{ }^{20}=$ 1.92 (c 0.55, $\left.\mathrm{CHCl}_{3}\right) ;{ }^{1} \mathrm{H}$ NMR $\left(\mathrm{CDCl}_{3}, 500 \mathrm{MHz}\right) \delta 1.23(\mathrm{~s}, 3 \mathrm{H}), 1.70-1.85(\mathrm{~m}, 3 \mathrm{H}), 1.86-$ $1.92(\mathrm{~m}, 1 \mathrm{H}), 2.07-2.16(\mathrm{~m}, 1 \mathrm{H}), 2.20-2.27(\mathrm{~m}, 1 \mathrm{H}), 5.75(\mathrm{dd}, J=4.6,3.2 \mathrm{~Hz}, 1 \mathrm{H}), 7.21-$ $7.45(\mathrm{~m}, 5 \mathrm{H}) \mathrm{ppm} ;{ }^{13} \mathrm{C}\left\{{ }^{1} \mathrm{H}\right\} \mathrm{NMR}\left(\mathrm{CDCl}_{3}, 125 \mathrm{MHz}\right) \delta 19.5,26.5,28.8,39.9,70.4,127.0$, 128.2, 129.1, 129.4, 141.8, 144.3 ppm; IR (NaCl) 3409, 3053, 3019, 2931, 2864, 1682, $1595,1490,1369,1179,1025 \mathrm{~cm}^{-1}$; HRMS calcd for $\mathrm{C}_{13} \mathrm{H}_{14}\left(\mathrm{M}-\mathrm{H}_{2} \mathrm{O}\right)^{+}: 170.1096$, found 170.1092 .

Preparation of 1-Ethyl-2-phenyl-cyclohex-2-enol (S8). General procedure A was applied to 2-phenylcyclohexen-1-one ${ }^{1}$ on a $86.2 \mathrm{mg}(0.5 \mathrm{mmol})$ scale, using $1.5 \mathrm{~mL}$ of diethylzinc solution. The crude product was purified by column chromatography on silica gel (hexanes / EtOAc : $95 / 5)$ to give $\mathbf{S 8}\left(40.5 \mathrm{mg}, 40 \%\right.$ yield, $95 \%$ ee) as an oil: $[\alpha]_{\mathrm{D}}{ }^{20}=$ $9.67\left(c\right.$ 0.60, $\left.\mathrm{CHCl}_{3}\right) ;{ }^{1} \mathrm{H} \mathrm{NMR}\left(\mathrm{CDCl}_{3}, 500 \mathrm{MHz}\right) \delta 0.65(\mathrm{t}, J=7.5 \mathrm{~Hz}, 3 \mathrm{H}), 1.40-1.47(\mathrm{~m}$, $1 \mathrm{H}), 1.50-1.56(\mathrm{~m}, 1 \mathrm{H}), 1.67-1.75(\mathrm{~m}, 5 \mathrm{H}), 2.0-2.07(\mathrm{~m}, 1 \mathrm{H}), 2.14-2.20(\mathrm{~m}, 1 \mathrm{H}), 5.78(\mathrm{dd}$, $J=5.2,2.5 \mathrm{~Hz}, 1 \mathrm{H}), 7.16-7.23(\mathrm{~m}, 3 \mathrm{H}), 7.35-7.38(\mathrm{~m}, 2 \mathrm{H}) \mathrm{ppm} ;{ }^{13} \mathrm{C}\left\{{ }^{1} \mathrm{H}\right\} \mathrm{NMR}\left(\mathrm{CDCl}_{3}\right.$, $125 \mathrm{MHz}) \delta 8.4,18.8,26.4,32.8,35.3,72.8,126.8,128.0,128.9,131.1,141.9,143.5$ ppm; IR $(\mathrm{NaCl}) 3459,3054,3019,2936,2866,1710,1599,1490,1439,1172 \mathrm{~cm}^{-1}$; HRMS calcd for $\mathrm{C}_{14} \mathrm{H}_{16}\left(\mathrm{M}-\mathrm{H}_{2} \mathrm{O}\right)^{+}:$184.1252, found 184.1254.

Preparation of 2-Benzylidene-1-methyl-cyclohexanol (S9). ${ }^{5}$ General procedure A was applied to 2-benzylidenecyclohexanone ${ }^{2}$ on a $93.2 \mathrm{mg}(0.5 \mathrm{mmol})$ scale, using $1.5 \mathrm{~mL}$ of dimethylzinc solution. The crude product was purified by column chromatography on silica gel (hexanes / EtOAc : 95 / 5) to give $\mathbf{S 9}$ (20 mg, 20\% yield, 99\% ee) as an oil: $[\alpha]_{\mathrm{D}}{ }^{20}$ $=+0.86\left(c 0.81, \mathrm{CH}_{2} \mathrm{Cl}_{2}\right)$. Spectral data for this compound is consistent with the previous charactarization. ${ }^{5}$

Preparation of 2-Benzylidene-1-ethyl-cyclohexanol (S10). General procedure A was applied to 2-benzylidenecyclohexanone ${ }^{2}$ on a $93.2 \mathrm{mg}(0.5 \mathrm{mmol})$ scale, using $1.5 \mathrm{~mL}$ 
of diethylzinc solution. The crude was purified by column chromatography on silica gel (hexanes / EtOAc : $95 / 5$ ) to give $\mathbf{S 1 0}$ (34.6 mg, 32\% yield, 99\% ee) as an oil: $[\alpha]_{\mathrm{D}}{ }^{20}=+$ $21.4\left(c\right.$ 1.40, $\left.\mathrm{CH}_{2} \mathrm{Cl}_{2}\right) ;{ }^{1} \mathrm{H}$ NMR $\left(\mathrm{C}_{6} \mathrm{D}_{6}, 500 \mathrm{MHz}\right) \delta 0.90(\mathrm{t}, J=7.4 \mathrm{~Hz}, 3 \mathrm{H}), 1.10-1.19$ (m, $1 \mathrm{H}), 1.22-1.35(\mathrm{~m}, 2 \mathrm{H}), 1.40-1.50(\mathrm{~m}, 3 \mathrm{H}), 1.62-1.74(\mathrm{~m}, 3 \mathrm{H}), 2.81-2.85(\mathrm{~m}, 1 \mathrm{H}), 6.78(\mathrm{~s}$, $1 \mathrm{H}), 7.06-7.25(\mathrm{~m}, 5 \mathrm{H}) \mathrm{ppm} ;{ }^{13} \mathrm{C}\left\{{ }^{1} \mathrm{H}\right\} \mathrm{NMR}\left(\mathrm{C}_{6} \mathrm{D}_{6}, 125 \mathrm{MHz}\right) \delta$ 7.6, 23.9, 27.2, 27.8, 30.6, 41.8, 75.1, 121.9, 126.4, 128.5, 129.5, 139.1, 146.4 ppm; IR (NaCl) 3430, 3078, 3035, $3021,2928,2855,1651,1598,1493,1445,1261,1163,1126 \mathrm{~cm}^{-1}$; HRMS calcd for $\mathrm{C}_{15} \mathrm{H}_{18}$ $\left(\mathrm{M}-\mathrm{H}_{2} \mathrm{O}\right)^{+}: 198.1409$, found 198.1409 .

Preparation of 1-Ethyl-cyclohex-2-enol (S11). ${ }^{6}$ General procedure A was applied to 2 -cyclohex-1-one on a $97 \mu \mathrm{L}(1.0 \mathrm{mmol})$ scale, using $3 \mathrm{~mL}$ of diethylzinc solution. The crude product was purified by column chromatography on silica gel (hexanes / EtOAc : 93 / 7) to give $\mathbf{S 1 1}\left(95 \mathrm{mg}, 75 \%\right.$ yield, $52 \%$ ee) as an oil: $[\alpha]_{\mathrm{D}}{ }^{20}=+48.7\left(c 1.55, \mathrm{CHCl}_{3}\right.$ ). Spectral data for this compound is consistent with the previous charactarization. ${ }^{6}$

Preparation of 1-Ethyl-3,5,5-trimethyl-cyclohex-2-enol (S12). General procedure A was applied to 3,5,5-Trimethyl-2-cyclohexen-1-one on a $150 \mu \mathrm{L}(1.0 \mathrm{mmol})$ scale, using $3 \mathrm{~mL}$ of diethylzinc solution. The crude product was purified by column chromatography on silica gel (hexanes / EtOAc : 95 / 5) to give $\mathbf{S 1 2}$ (84 mg, 50\% yield, 61\% ee) as an oil: $[\alpha]_{\mathrm{D}}{ }^{20}=+21.9\left(c 1.23, \mathrm{CH}_{3} \mathrm{OH}\right) ;{ }^{1} \mathrm{H}$ NMR $\left(\mathrm{C}_{6} \mathrm{D}_{6}, 500 \mathrm{MHz}\right) \delta 0.88(\mathrm{~s}, 3 \mathrm{H}), 0.92(\mathrm{t}, J=7.5$ $\mathrm{Hz}, 3 \mathrm{H}), 1.05$ (s, 3H), 1.32 (d, $J=14.0 \mathrm{~Hz}, 1 \mathrm{H}), 1.39-1.50$ (m, 4H), 1.55 (s, 3H), 1.59 (bs, $1 \mathrm{H}), 5.27$ (s, 1H) ppm; ${ }^{13} \mathrm{C}\left\{{ }^{1} \mathrm{H}\right\} \mathrm{NMR}\left(\mathrm{C}_{6} \mathrm{D}_{6}, 125 \mathrm{MHz}\right) \delta 8.1,24.1,27.5,30.1,32.0,37.3$, 44.8, 47.4, 71.2, 126.9, 134.8 ppm; IR (NaCl) 3389, 2949, 2824, 1673, 1453, 1376, 1364, $1177 \mathrm{~cm}^{-1}$; HRMS calcd for $\mathrm{C}_{11} \mathrm{H}_{18}\left(\mathrm{M}-\mathrm{H}_{2} \mathrm{O}\right)^{+}: 150.1408$, found 150.1362 .

\section{One-Pot Synthesis of Epoxy Alcohols.}

General Procedure B (Addition of $\mathbf{Z n M e}_{2}$ ). After carrying out the asymmetric dimethylzinc addition reaction, diethylzinc solution (1.0 $\mathrm{M}$ toluene solution, 1.5-2 equiv) 
was added to the reaction mixture at $0{ }^{\circ} \mathrm{C} . \mathrm{O}_{2}$ pressure was then applied from a balloon filled with $\mathrm{O}_{2}$. After stirring for $10 \mathrm{~min}$ the reaction mixture was warmed up to room temperature. When the epoxidation was complete, it was quenched with small amount of water $(\sim 2 \mathrm{~mL})$, dried over $\mathrm{MgSO}_{4}$, concentrated under reduced pressure, and purified by column chromatography on silica gel.

General Procedure $\mathbf{C}$ (Addition of $\mathbf{Z n E t}_{\mathbf{2}}$ ). Right after the asymmetric diethylzinc addition reaction was complete (tlc), $\mathrm{O}_{2}$ pressure was applied from a balloon at $0{ }^{\circ} \mathrm{C}$ and then the same procedure was followed as in general procedure B.

\section{Preparation of 1,2,5,5-Tetramethyl-7-oxa-bicyclo[4.1.0]heptan-2-ol (S13).}

General procedure B was applied to 2,4,4-trimethyl-2-cyclohexen-1-one on a $77 \mu \mathrm{L}(0.5$ mmol) scale. The crude product was purified by column chromatography on silica gel (hexanes / EtOAc : $85 / 15)$ to give $\mathbf{S 1 3}\left(70 \mathrm{mg}, 82 \%\right.$ yield, $99 \%$ ee) as an oil: $[\alpha]_{\mathrm{D}}{ }^{20}=-$ 3.07 (c 1.40, $\left.\mathrm{CHCl}_{3}\right) ;{ }^{1} \mathrm{H} \mathrm{NMR}\left(\mathrm{C}_{6} \mathrm{D}_{6}, 500 \mathrm{MHz}\right) \delta 0.73(\mathrm{~s}, 3 \mathrm{H}), 0.86-0.92(\mathrm{~m}, 1 \mathrm{H}), 0.99(\mathrm{~s}$, $3 \mathrm{H}), 1.14-1.19$ (m, 2H), 1.16 (s, 3H), 1.23 (s, 3H), 1.51-1.56 (m, 1H), 1.99 (bs, 1H), 2.47 (s, 1H) ppm; ${ }^{13} \mathrm{C}\left\{{ }^{1} \mathrm{H}\right\}$ NMR $\left(\mathrm{C}_{6} \mathrm{D}_{6}, 125 \mathrm{MHz}\right) \delta 18.3,24.9,26.3,26.9,29.8,33.0,34.9,64.2$, 70.4, 71.5 ppm; IR (NaCl) 3461, 2950, 2866, 1453, 1365, 1243, 1120, $1088 \mathrm{~cm}^{-1}$; HRMS calcd for $\mathrm{C}_{10} \mathrm{H}_{17} \mathrm{O}(\mathrm{M}-\mathrm{OH})^{+}: 153.1279$, found 153.1281 .

\section{Preparation of 2-Ethyl-1,5,5-trimethyl-7-oxa-bicyclo[4.1.0]heptan-2-ol (S14).}

General procedure $\mathrm{C}$ was applied to 2,4,4-trimethyl-2-cyclohexen-1-one on a $77 \mu \mathrm{L}(0.5$ mmol) scale. The crude product was purified by column chromatography on silica gel (hexanes / EtOAc : $90 / 10$ ) to give $\mathbf{S 1 4}\left(73 \mathrm{mg}, 80 \%\right.$ yield, $99 \%$ ee) as an oil: $[\alpha]_{\mathrm{D}}{ }^{20}=$ 5.27 (c 1.48, $\left.\mathrm{CHCl}_{3}\right) ;{ }^{1} \mathrm{H}$ NMR $\left(\mathrm{C}_{6} \mathrm{D}_{6}, 500 \mathrm{MHz}\right) \delta 0.74$ (s, 3H), 0.86 (ddd, $J=13.5,9.9$, $3.4 \mathrm{~Hz}, 1 \mathrm{H}), 0.92(\mathrm{t}, J=7.4 \mathrm{~Hz}, 3 \mathrm{H}), 1.01(\mathrm{~s}, 3 \mathrm{H}), 1.17$ (ddd, $J=13.5,9.9,3.4 \mathrm{~Hz}, 1 \mathrm{H})$, 1.23 (s, 3H), 1.25-1.33 (m, 2H), 1.43 (ddd, $J=13.5,9.9,3.4 \mathrm{~Hz}, 1 \mathrm{H}), 1.64-1.72(\mathrm{~m}, 1 \mathrm{H})$, 2.02 (bs, 1H), 2.47 (s, 1H) ppm; ${ }^{13} \mathrm{C}\left\{{ }^{1} \mathrm{H}\right\} \operatorname{NMR}\left(\mathrm{C}_{6} \mathrm{D}_{6}, 125 \mathrm{MHz}\right) \delta$ 7.9, 18.2, 26.6, 26.7, 
29.3, 29.5, 29.7, 32.5, 64.6, 71.5, 72.4 ppm; IR (NaCl) 3464, 2968, 2871, 1469, 1443, 1377, $1119,1064 \mathrm{~cm}^{-1}$; HRMS calcd for $\mathrm{C}_{11} \mathrm{H}_{21} \mathrm{O}_{2}(\mathrm{MH})^{+}: 185.1542$, found 185.1537 .

\section{Preparation of 1,2-Dimethyl-6-oxa-bicyclo[3.1.0]hexan-2-ol (S15). General} procedure B was applied to 2-methyl-2-cyclopenten-1-one on a $150 \mu \mathrm{L}(1.5 \mathrm{mmol})$ scale. The crude product was purified by column chromatography on silica gel (hexanes / EtOAc : $80 / 20$ ) to give $\mathbf{S 1 5}$ (96 mg, 50\% yield, $98 \%$ ee) as an oil: $[\alpha]_{\mathrm{D}}{ }^{20}=-22.6(c 1.64$, $\left.\mathrm{CHCl}_{3}\right) ;{ }^{1} \mathrm{H}$ NMR $\left(\mathrm{C}_{6} \mathrm{D}_{6}, 500 \mathrm{MHz}\right) \delta 0.99(\mathrm{~s}, 3 \mathrm{H}), 1.03-1.11(\mathrm{~m}, 1 \mathrm{H}), 1.23(\mathrm{~s}, 3 \mathrm{H}), 1.29$ (dd, $J=12.6,8.3 \mathrm{~Hz}, 1 \mathrm{H}), 1.42-1.47$ (m, $1 \mathrm{H}), 1.55-1.68$ (bs, $1 \mathrm{H}), 1.64$ (dd, $J=12.6,8.3 \mathrm{~Hz}$, 1H), $2.85(\mathrm{~s}, 1 \mathrm{H}) \mathrm{ppm} ;{ }^{13} \mathrm{C}\left\{{ }^{1} \mathrm{H}\right\} \operatorname{NMR}\left(\mathrm{C}_{6} \mathrm{D}_{6}, 125 \mathrm{MHz}\right) \delta 11.8,23.4,25.3,36.2,62.2,66.8$, 78.2 ppm; IR (NaCl) 3456, 2972, 1443, 1372, 1261, 1193, $1133 \mathrm{~cm}^{-1}$; HRMS calcd for $\mathrm{C}_{7} \mathrm{H}_{11} \mathrm{O}(\mathrm{M}-\mathrm{OH})^{+}: 111.0809$, found 111.0807 .

\section{Preparation of 2-Ethyl-1-methyl-6-oxa-bicyclo[3.1.0]hexan-2-ol (S16). General} procedure $\mathrm{C}$ was applied to 2-methyl-2-cyclopenten-1-one on a $100 \mu \mathrm{L}(1.0 \mathrm{mmol})$ scale. The crude product was purified by column chromatography on silica gel (hexanes / EtOAc : $85 / 15)$ to give $\mathbf{S 1 6}$ ( $85 \mathrm{mg}, 60 \%$ yield, $97 \%$ ee) as an oil: $[\alpha]_{\mathrm{D}}{ }^{20}=-8.19(c 1.16$, $\left.\mathrm{CHCl}_{3}\right) ;{ }^{1} \mathrm{H}$ NMR $\left(\mathrm{C}_{6} \mathrm{D}_{6}, 500 \mathrm{MHz}\right) \delta 0.80(\mathrm{t}, J=7.5 \mathrm{~Hz}, 3 \mathrm{H}), 1.10$ (dddd, $J=13.9,10.0$, 8.7, $1.2 \mathrm{~Hz}, 1 \mathrm{H}), 1.13-1.22(\mathrm{~m}, 1 \mathrm{H}), 1.23(\mathrm{~s}, 3 \mathrm{H}), 1.26-1.33(\mathrm{~m}, 1 \mathrm{H}), 1.50-1.58(\mathrm{~m}, 2 \mathrm{H})$, 1.65-1.73 (m, 2H), $2.90(\mathrm{~s}, 1 \mathrm{H}) \mathrm{ppm} ;{ }^{13} \mathrm{C}\left\{{ }^{1} \mathrm{H}\right\} \mathrm{NMR}\left(\mathrm{C}_{6} \mathrm{D}_{6}, 125 \mathrm{MHz}\right) \delta 7.9,12.1,25.9$, 28.9, 32.4, 62.8, 67.1, 80.7 ppm; IR (NaCl) 3457, 2967, 2936, 1440, 1375, 1182, $1123 \mathrm{~cm}^{-}$ ${ }^{1}$; HRMS calcd for $\mathrm{C}_{8} \mathrm{H}_{13} \mathrm{O}(\mathrm{M}-\mathrm{OH})^{+}:$125.0966, found 1125.0965 .

Preparation of 2-Methyl-1-pentyl-6-oxa-bicyclo[3.1.0]hexan-2-ol (S17). General procedure B was applied to 2-pentyl-2-cyclopenten-1-one on a $165 \mu \mathrm{L}(1.0 \mathrm{mmol})$ scale. The crude product was purified by column chromatography on silica gel (hexanes / EtOAc : $85 / 15)$ to give $\mathbf{S 1 7}\left(123 \mathrm{mg}, 67 \%\right.$ yield, $99 \%$ ee) as an oil: $[\alpha]_{\mathrm{D}}{ }^{20}=-12.9(c 1.05$, $\left.\mathrm{CHCl}_{3}\right) ;{ }^{1} \mathrm{H}$ NMR $\left(\mathrm{C}_{6} \mathrm{D}_{6}, 500 \mathrm{MHz}\right) \delta 0.89(\mathrm{t}, J=7.0 \mathrm{~Hz}, 3 \mathrm{H}), 1.07$ (s, 3H), 1.08-1.13 (m, $1 \mathrm{H}), 1.18-1.30(\mathrm{~m}, 5 \mathrm{H}), 1.34(\mathrm{dd}, J=12.6,8.3 \mathrm{~Hz}, 1 \mathrm{H}), 1.46-1.58(\mathrm{~m}, 3 \mathrm{H}), 1.65-1.71(\mathrm{~m}$, 
2H), 1.92-2.0 (m, 1H), $2.96(\mathrm{~s}, 1 \mathrm{H}) \mathrm{ppm} ;{ }^{13} \mathrm{C}\left\{{ }^{1} \mathrm{H}\right\}$ NMR $\left(\mathrm{C}_{6} \mathrm{D}_{6}, 125 \mathrm{MHz}\right) \delta 14.3,23.0$, 23.4, 24.5, 25.3, 25.7, 32.7, 36.9, 60.6, 69.3, 78.8 ppm; IR ( NaCl) 3456, 2934, 2861, 1462, $1362,1183,1132 \mathrm{~cm}^{-1}$; HRMS calcd for $\mathrm{C}_{11} \mathrm{H}_{20} \mathrm{O}_{2}(\mathrm{M})^{+}: 184.1463$, found 184.1464 .

Preparation of 2-Ethyl-1-pentyl-6-oxa-bicyclo[3.1.0]hexan-2-ol (S18). General procedure $\mathrm{C}$ was applied to 2-pentyl-2-cyclopenten-1-one on a $83 \mu \mathrm{L}(0.5 \mathrm{mmol})$ scale. The crude product was purified by column chromatography on silica gel (hexanes / EtOAc : 90 / 10) to give $\mathbf{S 1 8}\left(60 \mathrm{mg}, 60 \%\right.$ yield, $99 \%$ ee) as an oil: $[\alpha]_{\mathrm{D}}{ }^{20}=-5.18\left(c 0.83, \mathrm{CHCl}_{3}\right) ;{ }^{1} \mathrm{H}$ $\operatorname{NMR}\left(\mathrm{C}_{6} \mathrm{D}_{6}, 500 \mathrm{MHz}\right) \delta 0.82(\mathrm{t}, J=7.8 \mathrm{~Hz}, 3 \mathrm{H}), 0.88(\mathrm{t}, J=7.0 \mathrm{~Hz}, 3 \mathrm{H}), 1.07-1.12(\mathrm{~m}$, 1H), 1.18-1.35 (m, 7H), 1.46-1.73 (m, 6H), 1.97 (ddd, $J=14.3,10.5,4.2 \mathrm{~Hz}, 1 \mathrm{H}), 2.99$ (s, $1 \mathrm{H}), \mathrm{ppm} ;{ }^{13} \mathrm{C}\left\{{ }^{1} \mathrm{H}\right\} \mathrm{NMR}\left(\mathrm{C}_{6} \mathrm{D}_{6}, 125 \mathrm{MHz}\right) \delta 8.0,14.3,23.0,24.4,25.86,25.90,28.7,32.7$, 33.0, 61.2, 69.4, 81.3 ppm; IR (NaCl) 3480, 3016, 2956, 2859, 1709, 1463, 1378, 1176, $1120 \mathrm{~cm}^{-1}$; HRMS calcd for $\mathrm{C}_{12} \mathrm{H}_{22} \mathrm{O}_{2}(\mathrm{M})^{+}: 198.1620$, found 198.1625 .

Preparation of 4-Ethyl-2-phenyl-1-oxa-spiro[2.5]octan-4-ol (S19). General procedure $\mathrm{C}$ was applied to 2-benzylidenecyclohexanone ${ }^{2}$ on a $93.2 \mathrm{mg}(0.5 \mathrm{mmol})$ scale. The crude was purified by column chromatography on silica gel (hexanes / EtOAc : 95 / 5) to give $\mathbf{S 1 9}$ (40 mg, 34\% yield, 97\% ee) as an oil: $[\alpha]_{\mathrm{D}}{ }^{20}=+12.2\left(c 1.35, \mathrm{CH}_{2} \mathrm{Cl}_{2}\right) ;{ }^{1} \mathrm{H}$ $\operatorname{NMR}\left(\mathrm{C}_{6} \mathrm{D}_{6}, 500 \mathrm{MHz}\right) \delta 1.01(\mathrm{t}, J=7.4 \mathrm{~Hz}, 3 \mathrm{H}), 1.04-1.11(\mathrm{~m}, 2 \mathrm{H}), 1.22-1.24(\mathrm{~m}, 1 \mathrm{H})$, 1.24-1.32 (m, 1H), 1.36-1.47 (m, 3H), 1.48-1.54 (m, 1H), 1.55-1.64 (m, 1H), 1.75 -1.78 (m, 1H), 1.99 (bs, 1H), 4.42 (s, 1H), 7.01-7.05 (m, 1H), 7.08-7.14 (m, 2H), 7.24-7.27 (m, 2H) ppm; ${ }^{13} \mathrm{C}\left\{{ }^{1} \mathrm{H}\right\}$ NMR $\left(\mathrm{C}_{6} \mathrm{D}_{6}, 125 \mathrm{MHz}\right) \delta$ 7.7, 23.5, 23.7, 25.6, 27.9, 38.5, 60.1, 68.7, 71.4, 126.6, 127.5, 128.3, 136.7 ppm; IR (NaCl) 3489, 3087, 3061, 2932, 2961, 1644, 1604, 1498, 1450, 1377, 1172, $1151 \mathrm{~cm}^{-1}$; HRMS calcd for $\mathrm{C}_{15} \mathrm{H}_{20} \mathrm{O}_{2}(\mathrm{M})^{+}: 232.1463$, found 232.1461 . 


\section{Semipinacol Rearrangement Reaction of Epoxy Alcohols with $\mathrm{BF}_{3} \cdot \mathrm{OEt}_{2}$.}

General Procedure D. To a solution of epoxy alcohol $(\sim 0.5 \mathrm{mmol})$ in dry $\mathrm{CH}_{2} \mathrm{Cl}_{2}$ $(\sim 3 \mathrm{~mL})$ was added $\mathrm{BF}_{3} \cdot \mathrm{OEt}_{2}$ (2 equiv) under nitrogen at $-50{ }^{\circ} \mathrm{C}$. The mixture was warmed up to $0{ }^{\circ} \mathrm{C}$ for $2 \mathrm{~h}$. The reaction mixture was quenched with aqueous $\mathrm{NH}_{4} \mathrm{Cl}$ solution $(\sim 1$ $\mathrm{mL}$ ), extracted with $\mathrm{CH}_{2} \mathrm{Cl}_{2}$, the organic phase dried over $\mathrm{MgSO}_{4}$ and concentrated in vacuo. The residue was purified by column chromatography on silica gel to give the $\beta$ hydroxy ketone.

Preparation of 3-Hydroxy-2-methyl-2-pentyl-cyclopentanone (S20). General procedure D was applied to 2-methyl-1-pentyl-6-oxa-bicyclo[3.1.0]hexan-2-ol (S17) on a $86 \mathrm{mg}(0.47 \mathrm{mmol})$ scale. The crude product was purified by column chromatography on silica gel (hexanes / EtOAc : 80 / 20) to give S20 (60 mg, 70\% yield, 99\% ee) as an oil: $[\alpha]_{\mathrm{D}}{ }^{20}=+66.15\left(c 0.78, \mathrm{CHCl}_{3}\right) ;{ }^{1} \mathrm{H}$ NMR $\left(\mathrm{C}_{6} \mathrm{D}_{6}, 500 \mathrm{MHz}\right) \delta 0.77(\mathrm{~s}, 3 \mathrm{H}), 0.87(\mathrm{t}, J=7.1$ $\mathrm{Hz}, 3 \mathrm{H}), 1.15-1.34$ (m, 6H), 1.39 (ddd, $J=18.9,9.3,4.8 \mathrm{~Hz}, 1 \mathrm{H}), 1.49-1.63$ (m, 3H), 1.88 (ddd, $J=18.9,9.3,4.8 \mathrm{~Hz}, 1 \mathrm{H}), 2.17$ (ddd, $J=17.6,9.7,7.8 \mathrm{~Hz}, 1 \mathrm{H}), 3.52$ (s, 1H) ppm; ${ }^{13} \mathrm{C}\left\{{ }^{1} \mathrm{H}\right\}$ NMR $\left(\mathrm{C}_{6} \mathrm{D}_{6}, 125 \mathrm{MHz}\right) \delta 14.3,19.4,22.9,23.9,28.1,30.5,32.9,33.9,52.8,77.5$, 218.5 ppm; IR (NaCl) 3456, 2956, 2871, 1731, 1462, 1405, $1375 \mathrm{~cm}^{-1}$; HRMS calcd for $\mathrm{C}_{11} \mathrm{H}_{21} \mathrm{O}_{2}(\mathrm{MH})^{+}:$185.1542, found 185.1543 .

Preparation of 2-Ethyl-3-hydroxy-2-pentyl-cyclopentanone (S21). General procedure D was applied to 2-ethyl-1-pentyl-6-oxa-bicyclo[3.1.0]hexan-2-ol (S18) on a 80 $\mathrm{mg}(0.40 \mathrm{mmol})$ scale. The crude product was purified by column chromatography on silica gel (hexanes / EtOAc : $85 / 15)$ to give $\mathbf{S 2 1}\left(70 \mathrm{mg}, 89 \%\right.$ yield, $99 \%$ ee) as an oil: $[\alpha]_{\mathrm{D}}{ }^{20}=$ -3.54 (c 0.79, $\left.\mathrm{CHCl}_{3}\right) ;{ }^{1} \mathrm{H} \mathrm{NMR}\left(\mathrm{C}_{6} \mathrm{D}_{6}, 500 \mathrm{MHz}\right) \delta 0.78(\mathrm{t}, J=7.5 \mathrm{~Hz}, 3 \mathrm{H}), 0.87(\mathrm{t}, J=7.1$ $\mathrm{Hz}, 3 \mathrm{H}), 1.17-1.36$ (m, 9H), 1.42-1.50 (m, 1H), 1.52-1.61 (m, 2H), 1.65-1.73 (m, 1H), 1.83 $(\mathrm{ddd}, J=18.8,9.6,6.8 \mathrm{~Hz}, 1 \mathrm{H}), 2.16(\mathrm{ddd}, J=18.8,9.6,6.8 \mathrm{~Hz}, 1 \mathrm{H}), 3.72(\mathrm{t}, J=5.1 \mathrm{~Hz}$, 1H) ppm; ${ }^{13} \mathrm{C}\left\{{ }^{1} \mathrm{H}\right\} \operatorname{NMR}\left(\mathrm{C}_{6} \mathrm{D}_{6}, 125 \mathrm{MHz}\right) \delta 8.3,14.3,23.0,23.7,25.0,27.4,28.4,33.1$, 
34.7, 56.3, 76.1, 218.3 ppm; IR ( $\mathrm{NaCl}) 3446,2956,2871,1725,1459,1406,1380,1111$ $\mathrm{cm}^{-1}$; HRMS calcd for $\mathrm{C}_{12} \mathrm{H}_{22} \mathrm{O}_{2}+\mathrm{Na}(\mathrm{M}+\mathrm{Na})^{+}: 221.1517$, found 221.1507 .

Preparation of 3-Hydroxy-2,2-dimethyl-cyclopentanone $\quad$ (S22). ${ }^{7} \quad$ General procedure D was applied to 1,2-dimethyl-6-oxa-bicyclo[3.1.0]hexan-2-ol (S15) on a $80 \mathrm{mg}$ (0.62 mmol) scale. The crude was purified by column chromatography on silica gel (hexanes / EtOAc : $75 / 25)$ to give $\mathbf{S 2 2}\left(60 \mathrm{mg}, 75 \%\right.$ yield, $98 \%$ ee) as an oil: $[\alpha]_{\mathrm{D}}{ }^{20}=$ $+7.47\left(c 0.75, \mathrm{CHCl}_{3}\right)$. Spectral data for this compound is consistent with the previous charactarization. $^{7}$

Preparation of 2-Ethyl-3-hydroxy-2-methyl-cyclopentanone (S23). General procedure D was applied to 2-Ethyl-1-methyl-6-oxa-bicyclo[3.1.0]hexan-2-ol on a $57 \mathrm{mg}$ $(0.4 \mathrm{mmol})$ scale. The crude was purified by column chromatography on silica gel (hexanes / EtOAc : $80 / 20)$ to give $\mathbf{S 2 3}$ (41 mg, 73\% yield, $97 \%$ ee) as an oil: $[\alpha]_{\mathrm{D}}{ }^{20}=-48.0(c 0.81$, $\left.\mathrm{CHCl}_{3}\right) ;{ }^{1} \mathrm{H}$ NMR $\left(\mathrm{C}_{6} \mathrm{D}_{6}, 500 \mathrm{MHz}\right) \delta 0.73(\mathrm{t}, J=7.5 \mathrm{~Hz}, 3 \mathrm{H}), 0.92(\mathrm{~s}, 3 \mathrm{H}), 1.18-1.29(\mathrm{~m}$, 3H), 1.34-1.44 (m, 1H), 1.62-1.68 (m, 1H), 1.75 (ddd, $J=18.7,9.3,7.0 \mathrm{~Hz}, 1 \mathrm{H}), 2.14$ (ddd, $J=18.5,9.5,5.4 \mathrm{~Hz}, 1 \mathrm{H}), 3.64(\mathrm{t}, J=5.6 \mathrm{~Hz}, 1 \mathrm{H}) \mathrm{ppm} ;{ }^{13} \mathrm{C}\left\{{ }^{1} \mathrm{H}\right\} \operatorname{NMR}\left(\mathrm{C}_{6} \mathrm{D}_{6}, 125 \mathrm{MHz}\right) \delta$ 8.5, 14.6, 28.0, 28.1, 34.7, 53.3, 75.3, 218.8 ppm; IR (NaCl) 3436, 2969, 2936, 1734, 1458, $1406 \mathrm{~cm}^{-1}$; HRMS calcd for $\mathrm{C}_{8} \mathrm{H}_{15} \mathrm{O}_{2}(\mathrm{MH})^{+}:$143.1072, found 143.1079 .

\section{Conditions for the Determination of Enantiomeric Excesses.}

The racemic alcohols were prepared by addition of Grignard reagents to the corresponding ketones. The racemic epoxy alcohols were prepared by $m$ CPBA epoxidation method using the racemic alcohols. Due to the sensitivity of tertiary alcohols S3-S6, these product were converted to the $\beta$-hydroxy ketones which were transformed into the trifluoroacetate before ee determination by chiral capillary GC. The tertiary alcohols $\mathbf{S 1}, \mathbf{S 2}$, S11, S12 and the epoxy alcohols S13, S14 were analyzed directly by chiral capillary GC. The epoxy alcohols S15-S18 were treated with $\mathrm{BF}_{3} \cdot \mathrm{OEt}_{2}$ to the $\beta$-hydroxy ketones and 
analyzed by chiral capillary GC, therefore, the ee of $\beta$-hydroxy ketones S20-S23 must be the same as that of the corresponding epoxy alcohols S15-S18. The specifications for the GC analyses were as follows: Fused silica chiral capillary column (Supelco $\beta$-Dex 120): 30 $\mathrm{m} \times 0.25 \mathrm{~mm}$ (id) x $0.25 \mu \mathrm{m}$ film thickness. Carrier gas: nitrogen. Inlet temperature: $250{ }^{\circ} \mathrm{C}$. Detector: FID, $270{ }^{\circ} \mathrm{C}$. The conditions for the resolution of the racemates by $\mathrm{GC}$ are given below.

1,2,4,4-Tetramethyl-cyclohex-2-enol (S1). $t_{1}=15.5 \mathrm{~min}, \mathrm{t}_{2}=16.7 \mathrm{~min}\left(110{ }^{\circ} \mathrm{C}\right.$, $1.6 \mathrm{~mL} / \mathrm{min})$.

1-Ethyl-2,4,4-trimethyl-cyclohex-2-enol (S2). $\mathrm{t}_{1}=10.5 \mathrm{~min}, \mathrm{t}_{2}=11.2 \mathrm{~min}\left(110{ }^{\circ} \mathrm{C}\right.$, $1.6 \mathrm{~mL} / \mathrm{min})$.

1,2-Dimethyl-cyclopent-2-enol (S3) \{after epoxidation, rearrangement, and derivatization $\} . \mathrm{t}_{1}=29.8 \mathrm{~min}, \mathrm{t}_{2}=32.4 \mathrm{~min}\left(70{ }^{\circ} \mathrm{C}, 1.5 \mathrm{~mL} / \mathrm{min}\right)$.

1-Ethyl-2-methyl-cyclopent-2enol (S4) \{after epoxidation, rearrangement, and derivatization $\} . \mathrm{t}_{1}=51.2 \mathrm{~min}, \mathrm{t}_{2}=52.7 \mathrm{~min}\left(70{ }^{\circ} \mathrm{C}, 1.5 \mathrm{~mL} / \mathrm{min}\right)$.

1-Methyl-2-pentyl-cyclopent-2-enol (S5) \{after epoxidation, rearrangement, and derivatization $\} . \mathrm{t}_{1}=24.6 \mathrm{~min}, \mathrm{t}_{2}=27.0 \mathrm{~min}\left(110{ }^{\circ} \mathrm{C}, 1.6 \mathrm{~mL} / \mathrm{min}\right)$.

1-Ethyl-2-pentyl-cyclopent-2-enol (S6) \{after epoxidation, rearrnagement, and derivatization $\} . \mathrm{t}_{1}=32.5 \mathrm{~min}, \mathrm{t}_{2}=34.4 \mathrm{~min}\left(110{ }^{\circ} \mathrm{C}, 1.6 \mathrm{~mL} / \mathrm{min}\right)$.

1-Ethyl-cyclohex-2-enol (S11). $\mathrm{t}_{1}=144.7 \mathrm{~min}, \mathrm{t}_{2}=152.5 \mathrm{~min}\left(60{ }^{\circ} \mathrm{C}, 1.5 \mathrm{~mL} / \mathrm{min}\right)$.

1-Ethyl-3,5,5-trimethyl-cyclohex-2-enol (S12). $\mathrm{t}_{1}=63.8 \mathrm{~min}, \mathrm{t}_{2}=67.4 \mathrm{~min}\left(80{ }^{\circ} \mathrm{C}\right.$, $1.7 \mathrm{~mL} / \mathrm{min})$.

1,2,5,5-Tetramethyl-7-oxa-bycyclo[4.1.0]heptan-2-ol (S13). $\mathrm{t}_{1}=10.5 \mathrm{~min}, \mathrm{t}_{2}=11.2 \mathrm{~min}$ $\left(110^{\circ} \mathrm{C}, 1.6 \mathrm{~mL} / \mathrm{min}\right)$.

2-Ethyl-1,5,5-trimethyl-7-oxa-bycyclo[4.1.0]heptan-2-ol (S14). $t_{1}=27.2 \mathrm{~min}, \mathrm{t}_{2}=30.5$ $\min \left(110^{\circ} \mathrm{C}, 1.6 \mathrm{~mL} / \mathrm{min}\right)$.

1,2-Dimethyl-6-oxa-bicyclo[3.1.0]hexan-2-ol (S15) \{after rearrangement and derivatization $\} . \mathrm{t}_{1}=29.8 \mathrm{~min}, \mathrm{t}_{2}=32.4 \mathrm{~min}\left(70{ }^{\circ} \mathrm{C}, 1.5 \mathrm{~mL} / \mathrm{min}\right)$.

2-Ethyl-1-methyl-6-oxa-bicyclo[3.1.0]hexan-2-ol (S16) \{after rearrangement and derivatization $\} . \mathrm{t}_{1}=51.2 \mathrm{~min}, \mathrm{t}_{2}=52.7 \mathrm{~min}\left(70{ }^{\circ} \mathrm{C}, 1.5 \mathrm{~mL} / \mathrm{min}\right)$. 
2-Methyl-1-pentyl-6-oxa-bicyclo[3.1.0]hexan-2-ol (S17) \{after rearrangement and derivatization $\} . \mathrm{t}_{1}=24.6 \mathrm{~min}, \mathrm{t}_{2}=27.0 \mathrm{~min}\left(110{ }^{\circ} \mathrm{C}, 1.6 \mathrm{~mL} / \mathrm{min}\right)$.

2-Ethyl-1-pentyl-6-oxa-bicyclo[3.1.0]hexan-2-ol (S18) \{after rearrangement and derivatization $\} . \mathrm{t}_{1}=32.5 \mathrm{~min}, \mathrm{t}_{2}=34.4 \mathrm{~min}\left(110^{\circ} \mathrm{C}, 1.6 \mathrm{~mL} / \mathrm{min}\right)$.

3-Hydroxy-2-methyl-2-pentyl-cyclopentanone (S20) \{after rearrangement and derivatization\}. The same as $\mathbf{S 1 7}$.

2-Ethyl-3-hydroxy-2-pentyl-cyclopentanone (S21) \{after rearrangement and derivatization\}. The same as S18.

3-Hydroxy-2,2-dimethyl-cyclopentanone (S22) \{after rearrangement and derivatization\}. The same as $\mathbf{S 1 5}$.

2-Ethyl-3-hydroxy-2-methyl-cyclopentanone (S23) \{after rearrangement and derivatization\}. The same as S16.

Chiral HPLC analyses of S7-S10 were performed using a Chiralcel OD-H column. The analysis of $\mathbf{S 1 9}$ was performed using a Chiralpak AS column.

1-Methyl-2-phenyl-cyclohex-2-enol (S7). $t_{1}=7.7 \mathrm{~min}, \mathrm{t}_{2}=10.2 \mathrm{~min}$ (hexane $/ 2$ propanol : 95 / 5, $0.7 \mathrm{~mL} / \mathrm{min})$.

1-Ethyl-2-phenyl-cyclohex-2-enol (S8). $\mathrm{t}_{1}=7.9 \mathrm{~min}, \mathrm{t}_{2}=10.6 \mathrm{~min}$ (hexane $/ 2$-propanol : $95 / 5,0.7 \mathrm{~mL} / \mathrm{min})$.

2-Benzylidene-1-methyl-cyclohexanol (S9). $\mathrm{t}_{1}=12.9 \mathrm{~min}, \mathrm{t}_{2}=15.1 \mathrm{~min}$ (hexane $/ 2$ propanol : 95 / 5, $0.7 \mathrm{~mL} / \mathrm{min})$.

2-Benzylidene-1-ethyl-cyclohexanol (S10). $t_{1}=10.4 \mathrm{~min}, \mathrm{t}_{2}=12.3 \mathrm{~min}$ (hexane $/ 2$ propanol : 95 / 5, $0.7 \mathrm{~mL} / \mathrm{min})$.

4-Ethyl-2-phenyl-1-oxa-spiro[2.5]octan-4-ol (S19). $\mathrm{t}_{1}=12.6 \mathrm{~min}, \mathrm{t}_{2}=16.5 \mathrm{~min}$ (hexane / 2-propanol : 97 / 3, $0.4 \mathrm{~mL} / \mathrm{min})$. 


\section{References.}

1) Chidambaram, N.; Chandrasekaran, S. J. Org. Chem, 1987, 52, 5048-5051.

2) Fogliato, G.; Fronza, G.; Fuganti, C.; Lanati, S.; Rallo, R.; Figoni, R.; Servi, S. Tetrahedron 1995, 51, 10231-10240.

3) Mironov, V. A.; Akhrem, A. A. Izv. Akad. Nauk SSSR, Ser. Khim. 1973, 38.

4) Trost, B. M.; Pinkerton, A. B. J. Org. Chem. 2001, 66, 7714-7722.

5) Shim, S. C.; Hwang, J. T.; Kang, H.-Y.; Chang, M. H. Tetrahedron Lett. 1990, 31, 47654768.

6) Alonso, E.; Ramon, D. J.; Yus, M. Tetrahedron, 1996, 52, 14341-14348.

7) Shimizu, M.; Yamada, S.; Fujita, Y.; Kobayashi, F. Tetrahedron: Asymmetry 2000, 11, 3883-3886. 


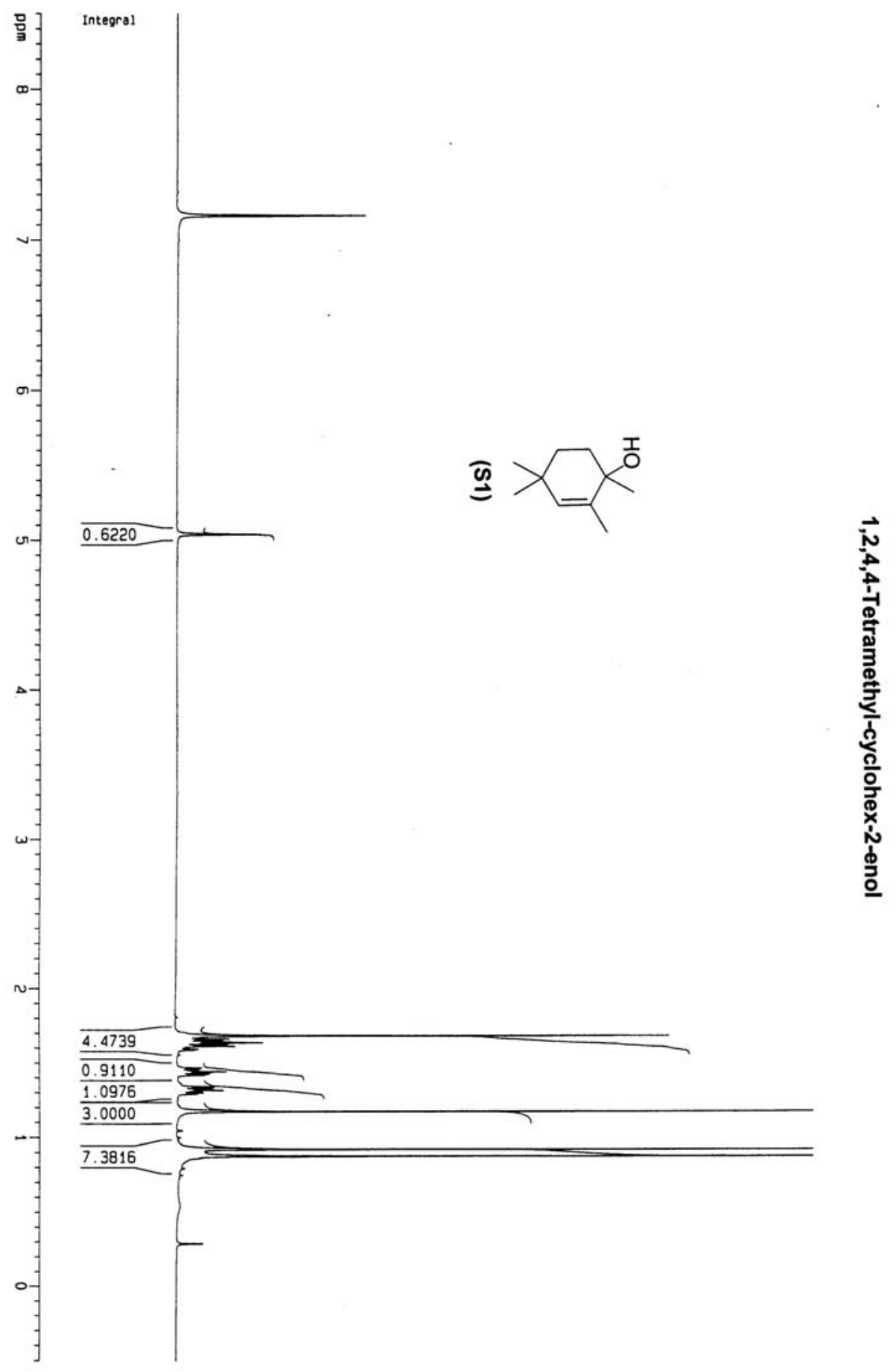




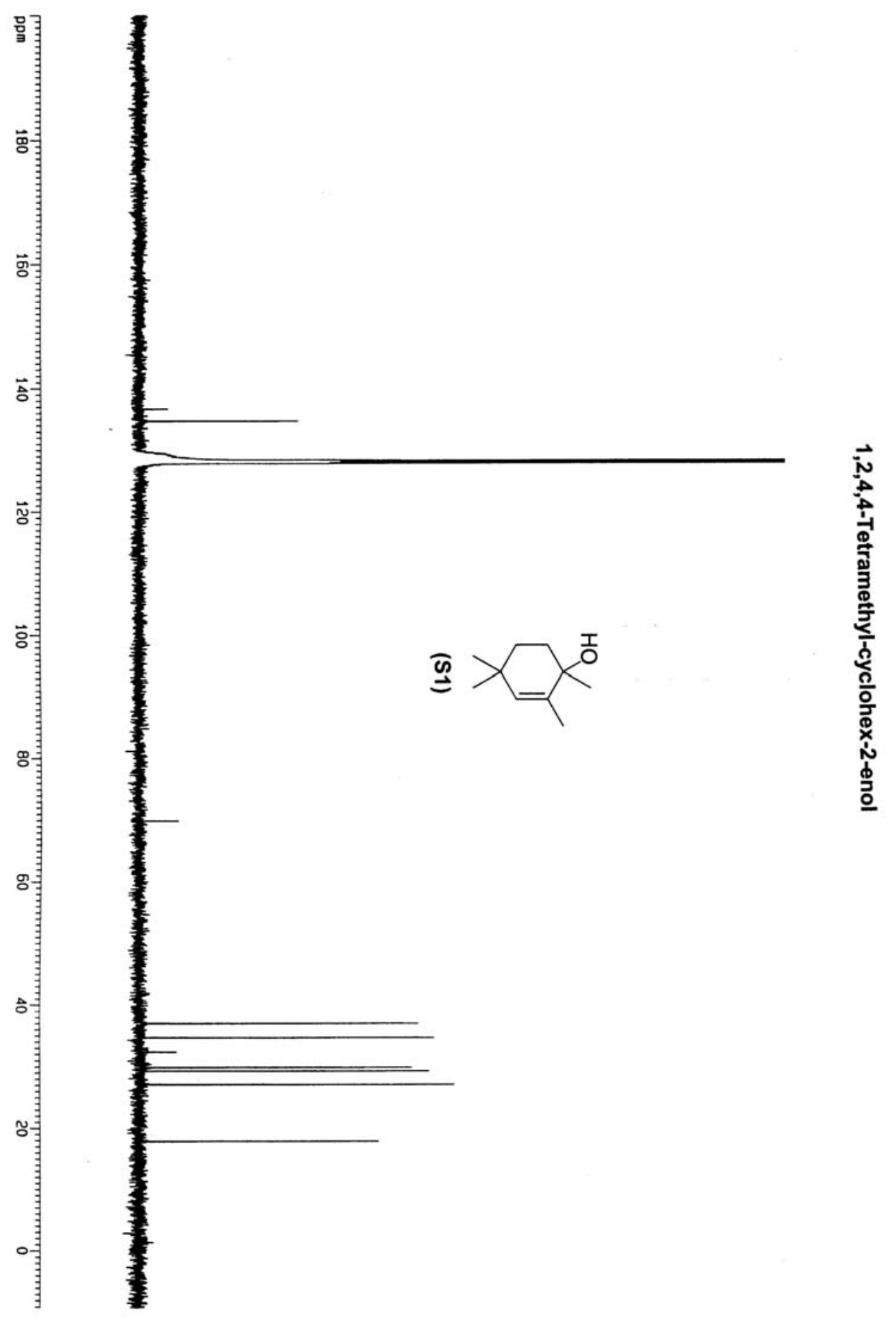




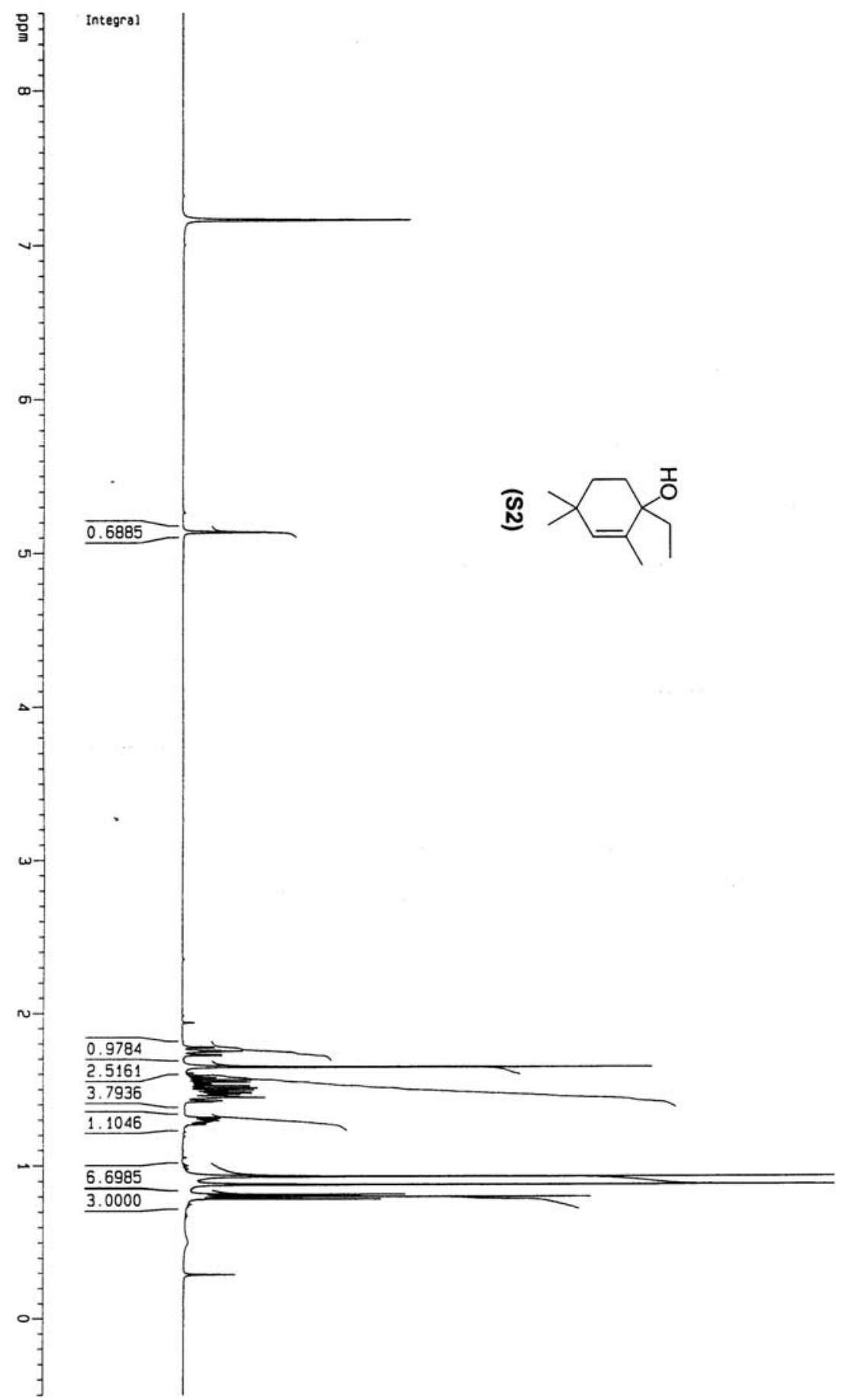

旁 


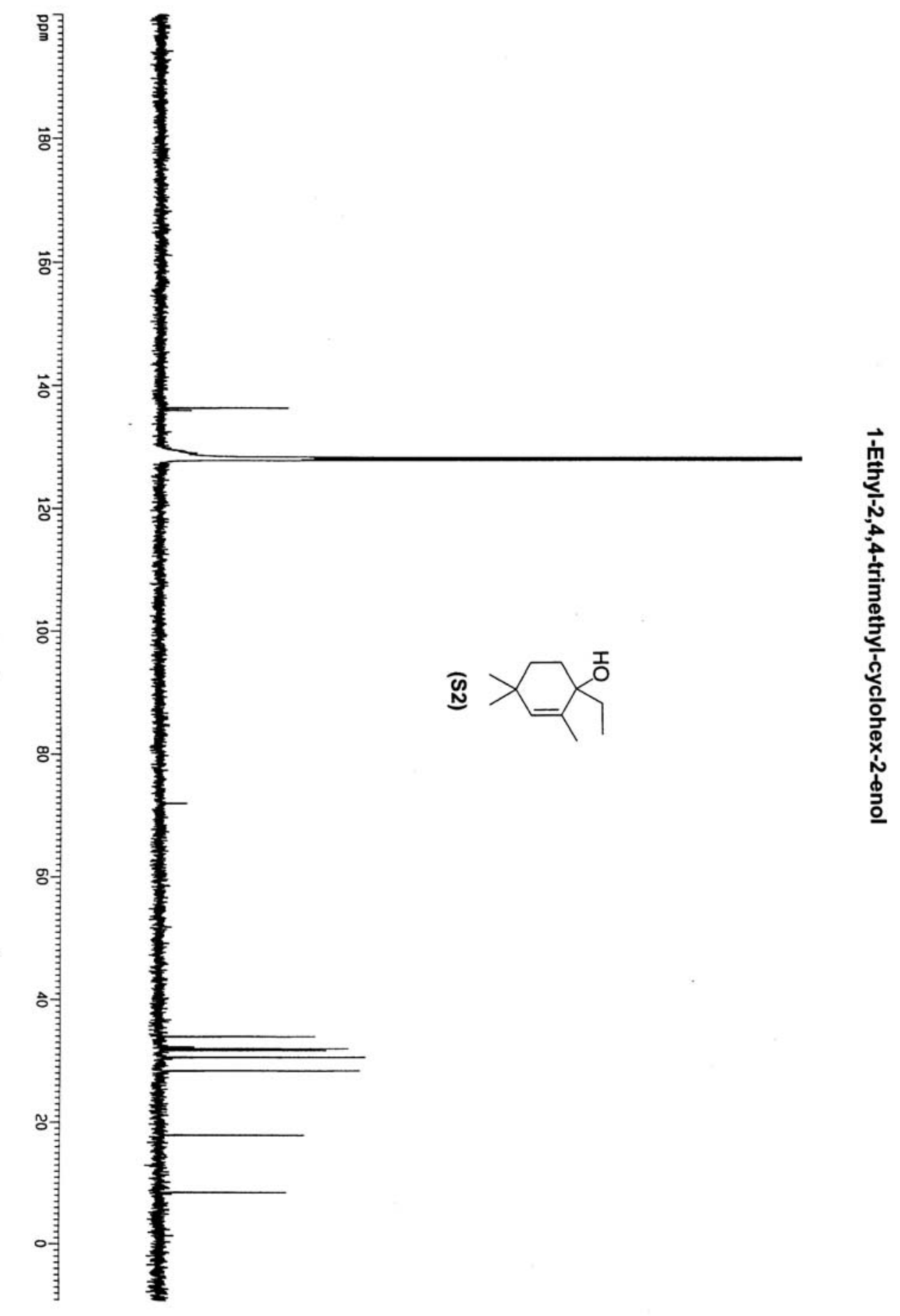




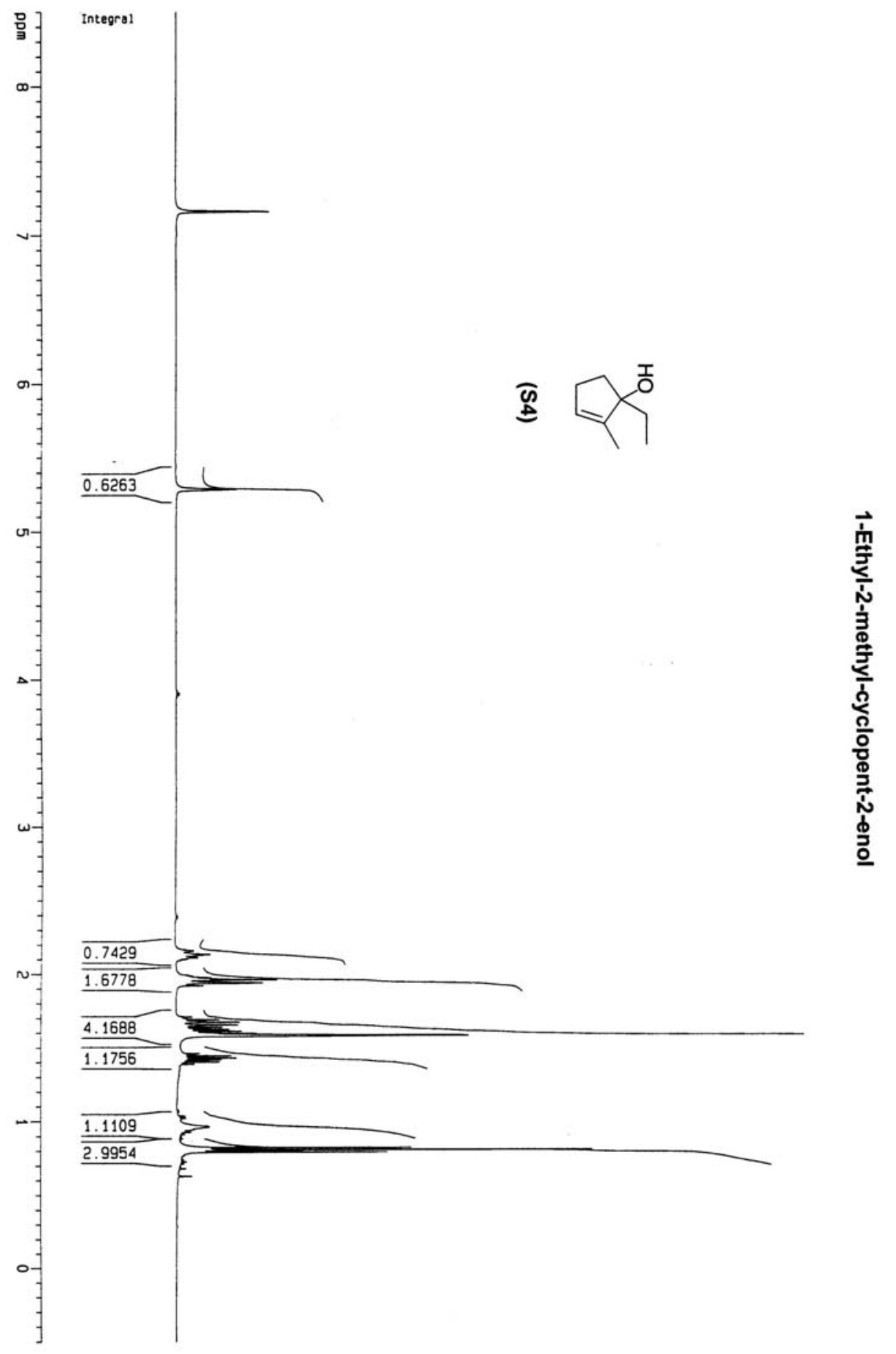




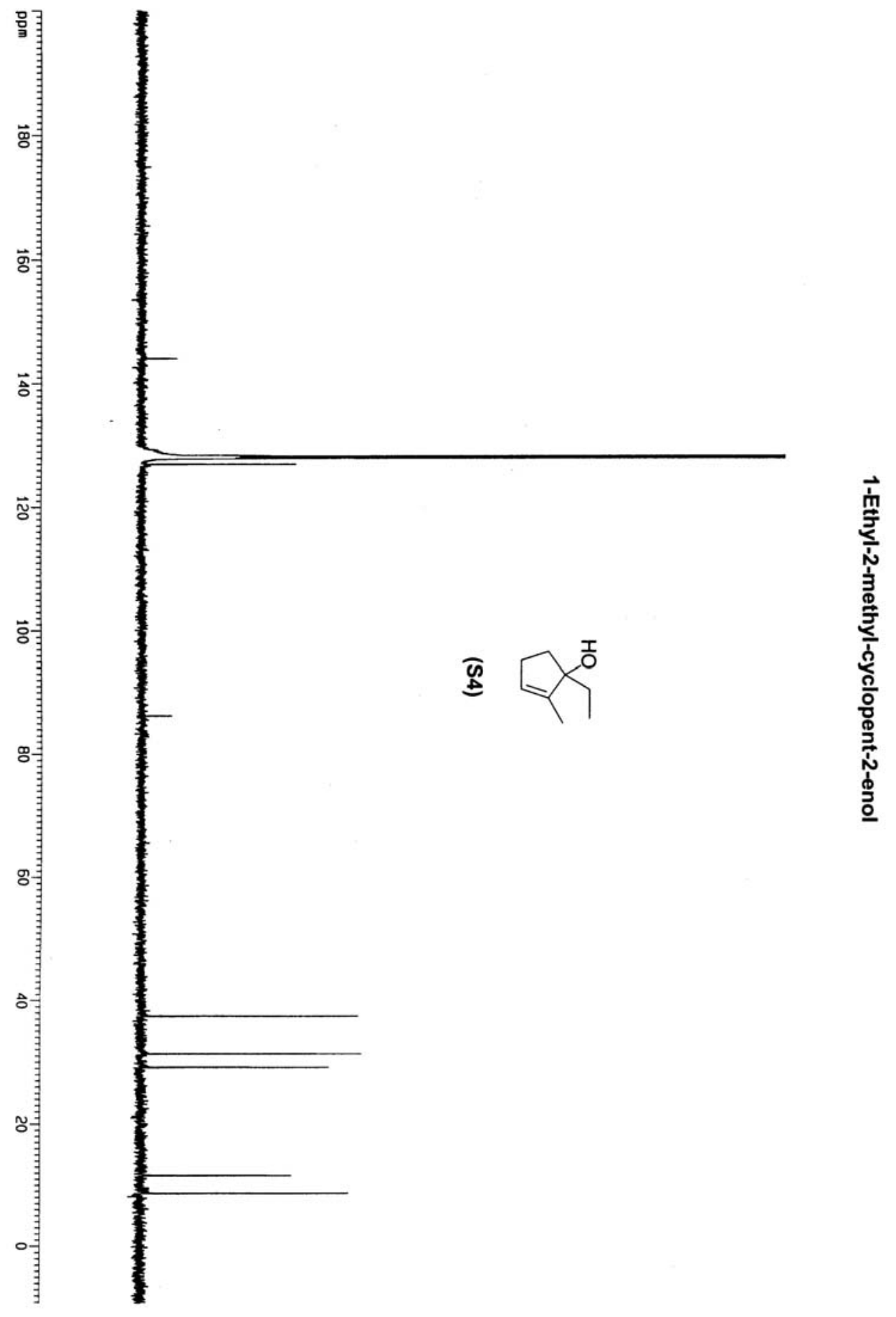




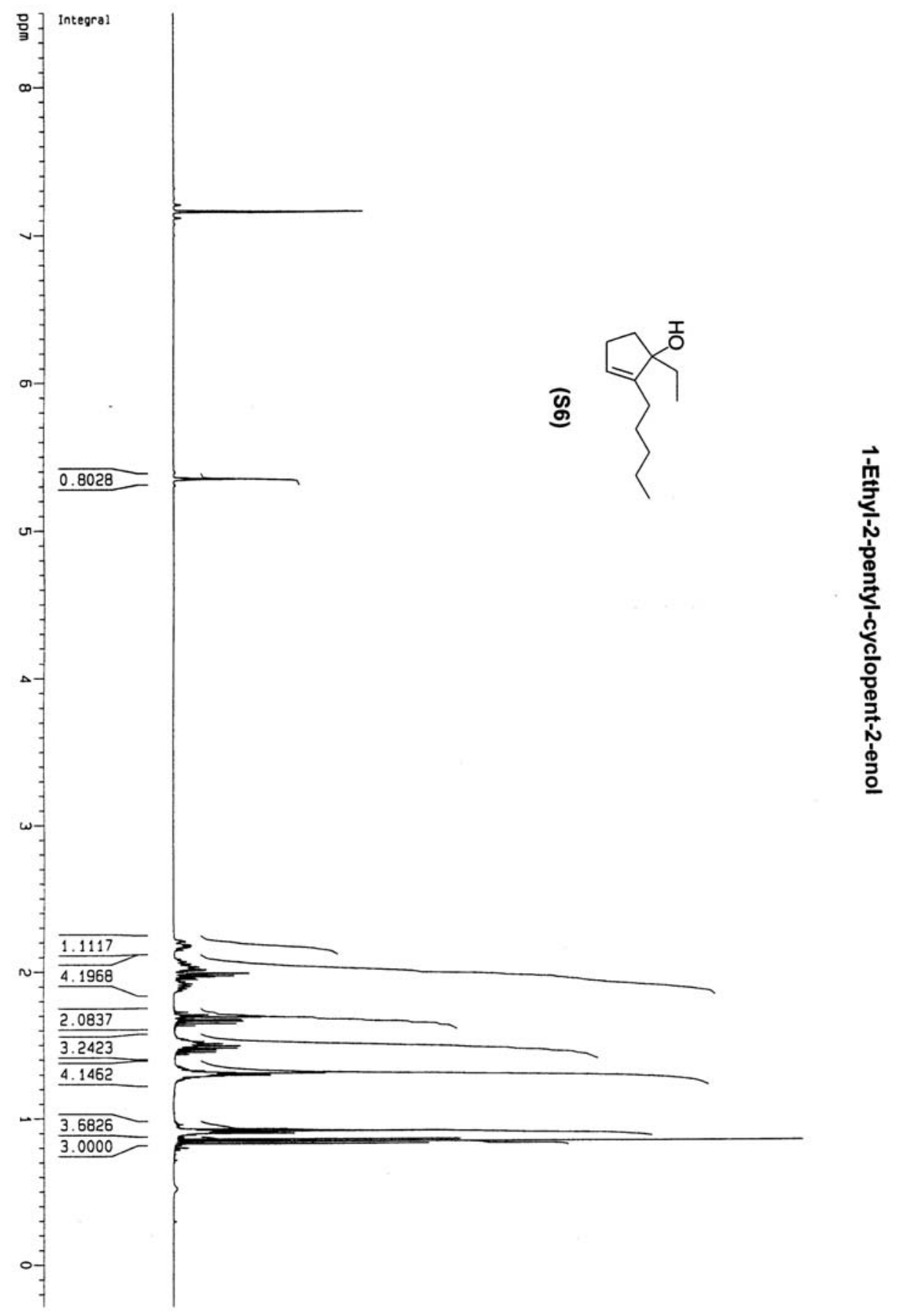




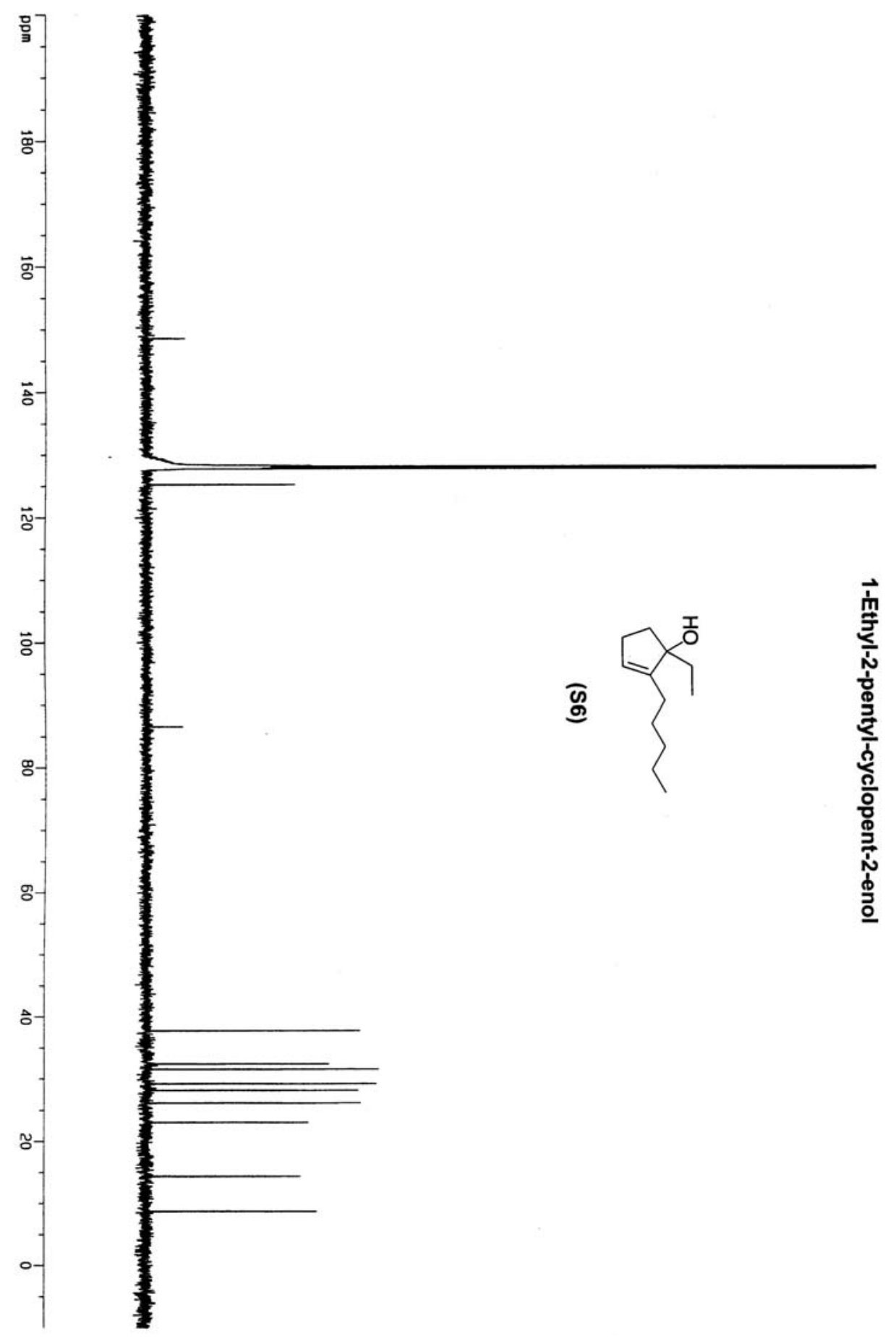




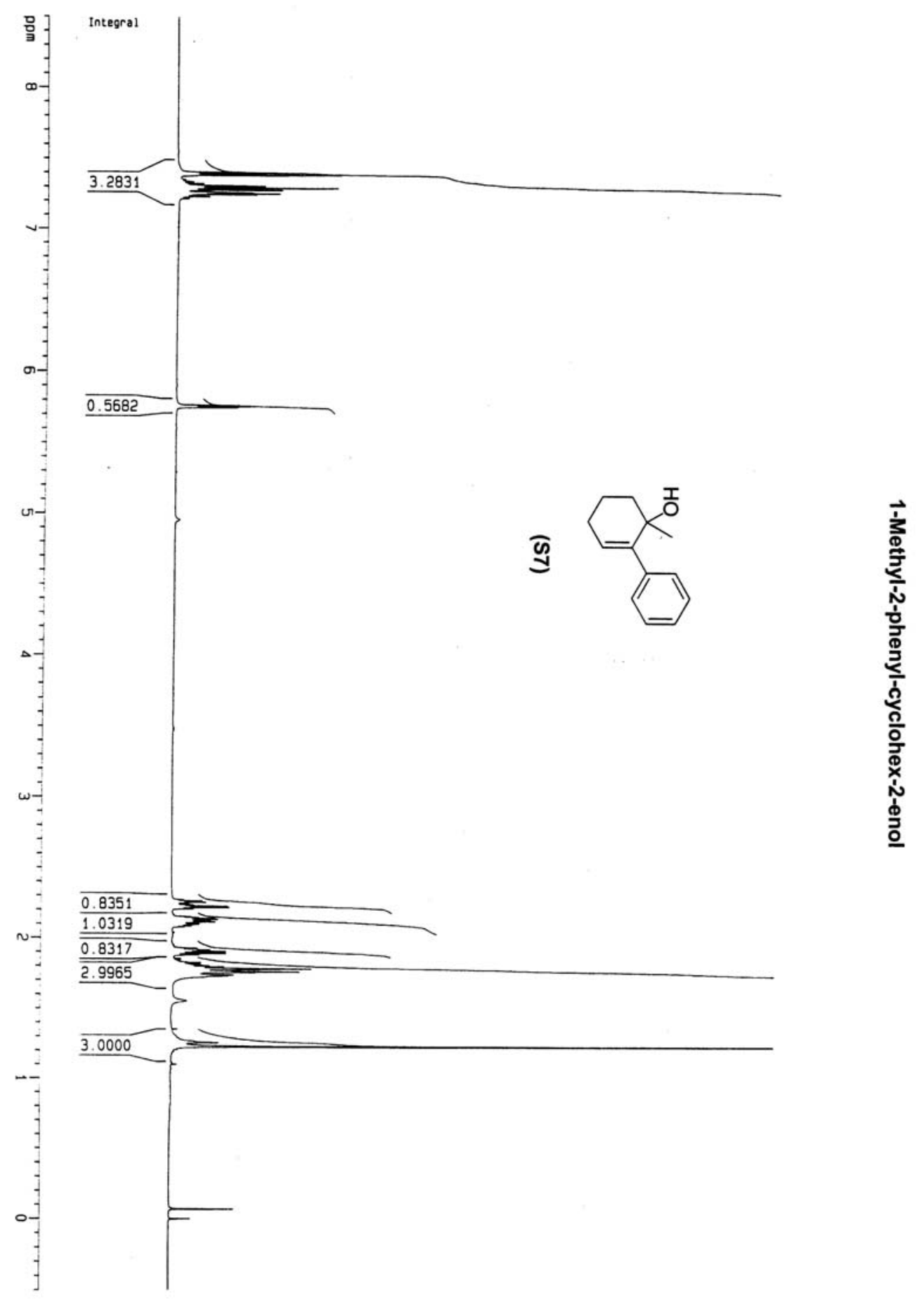




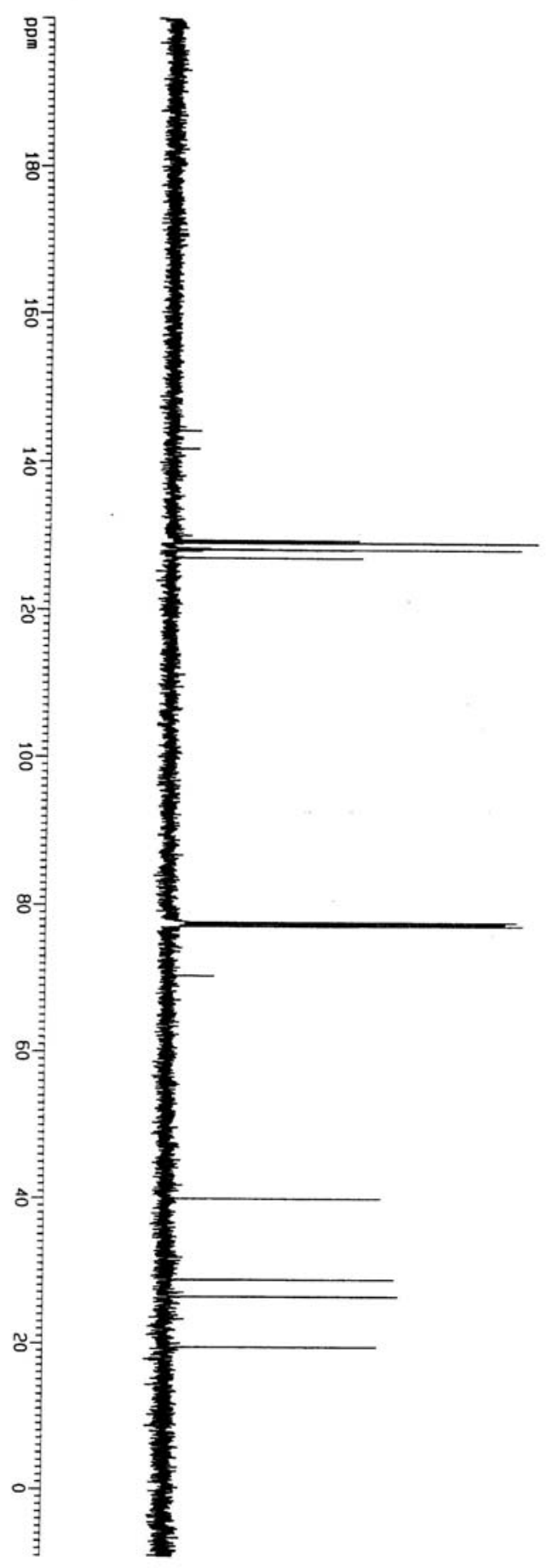

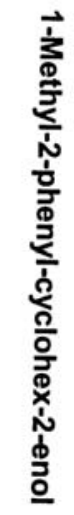

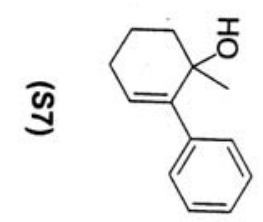




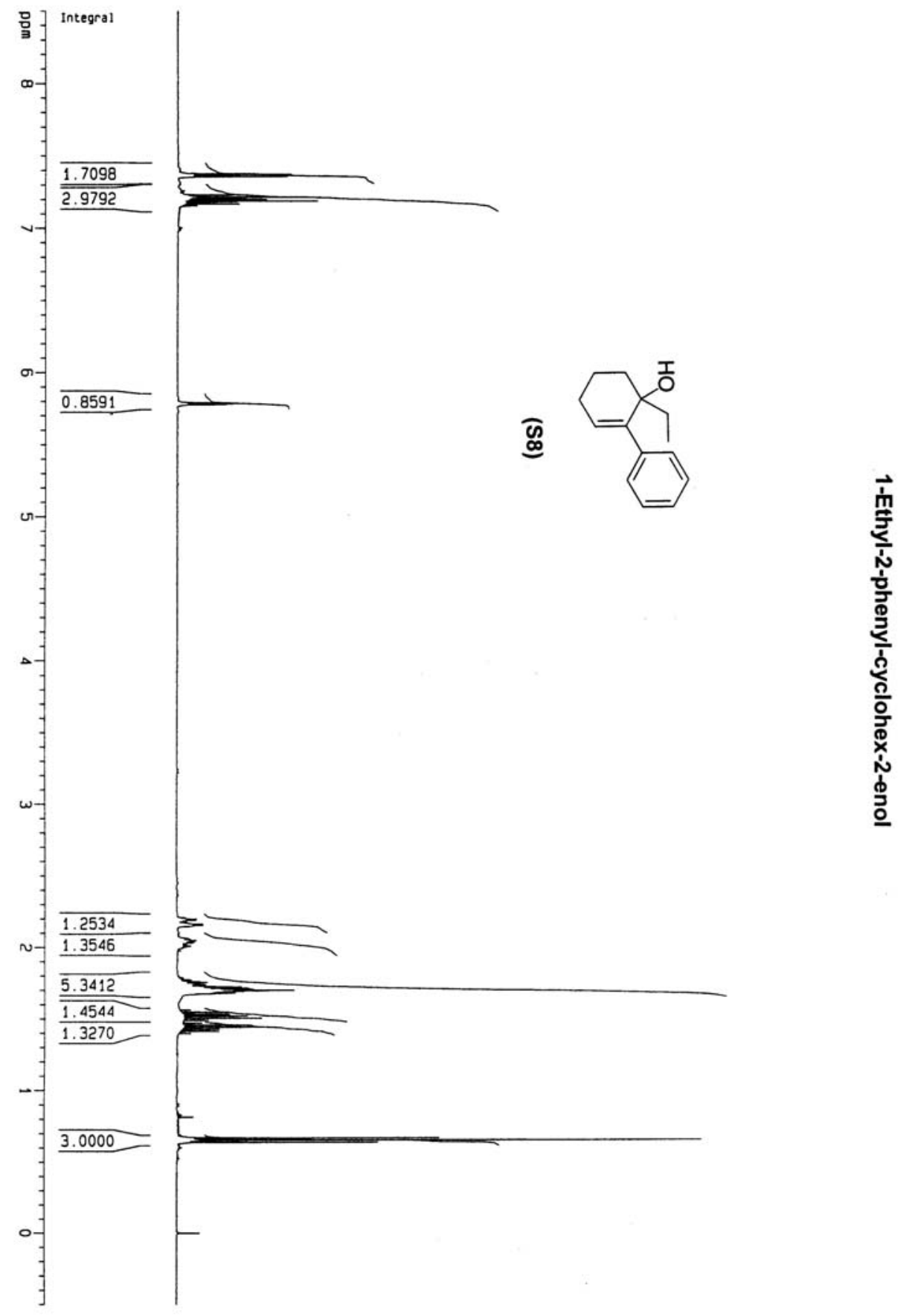



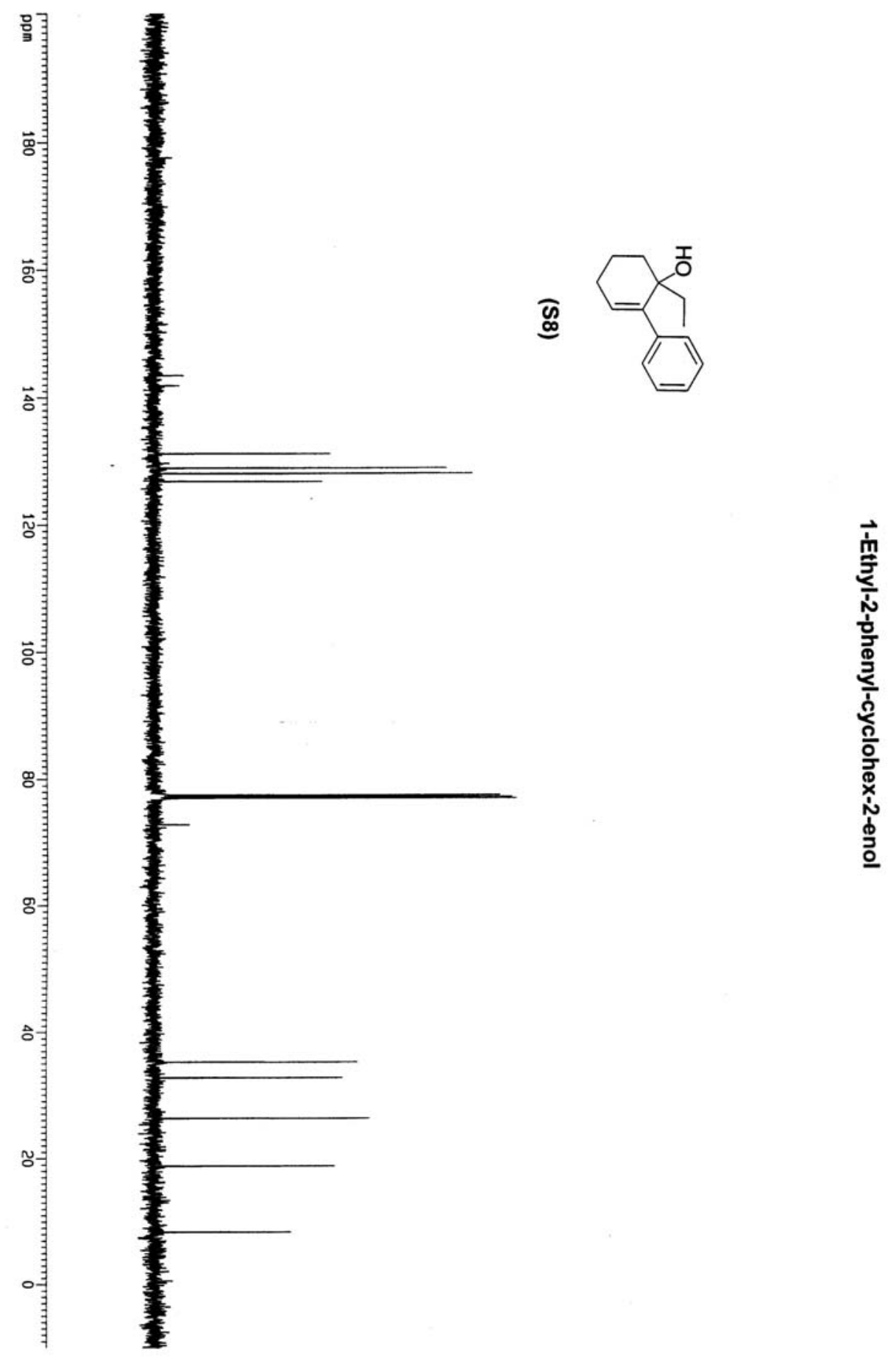


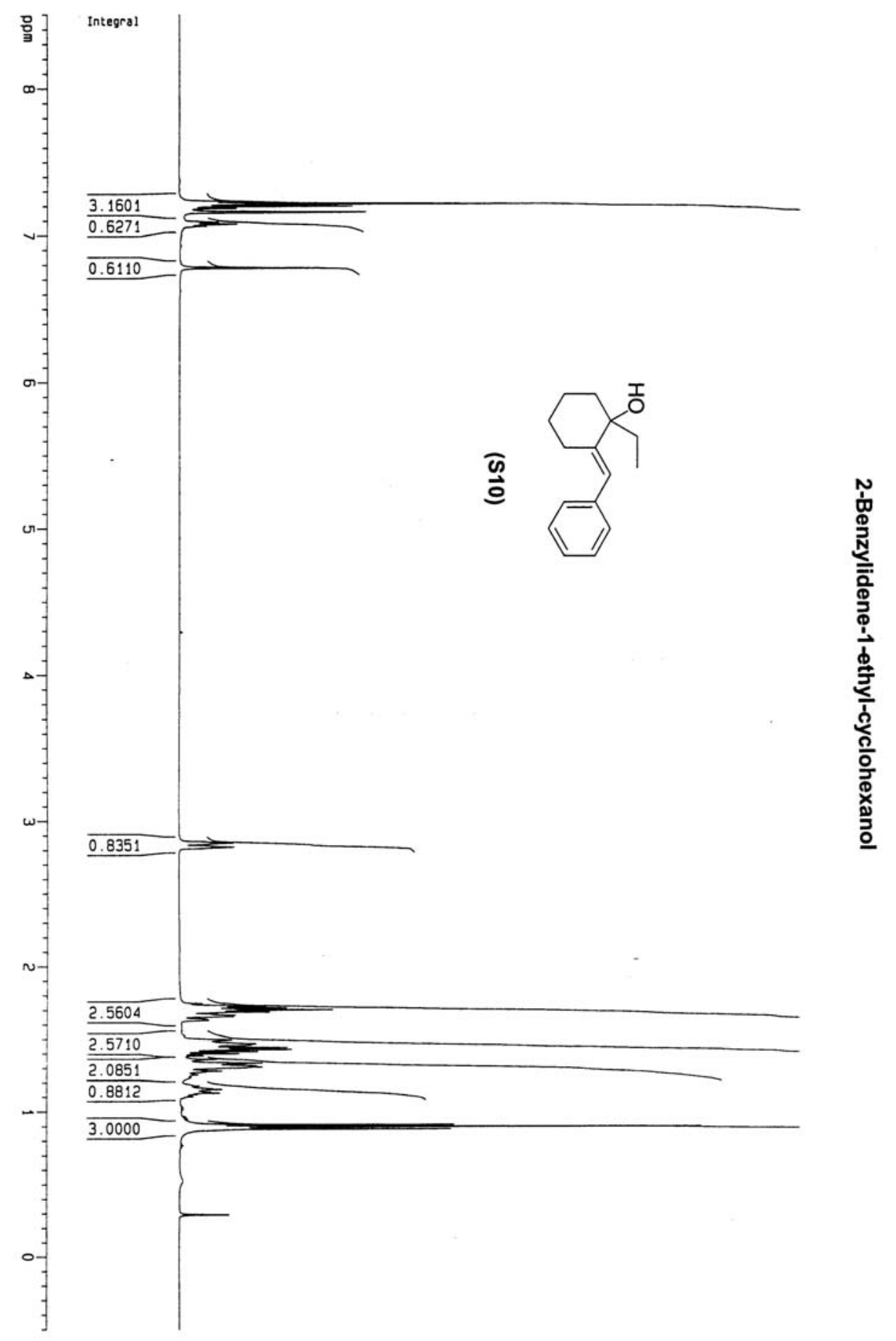




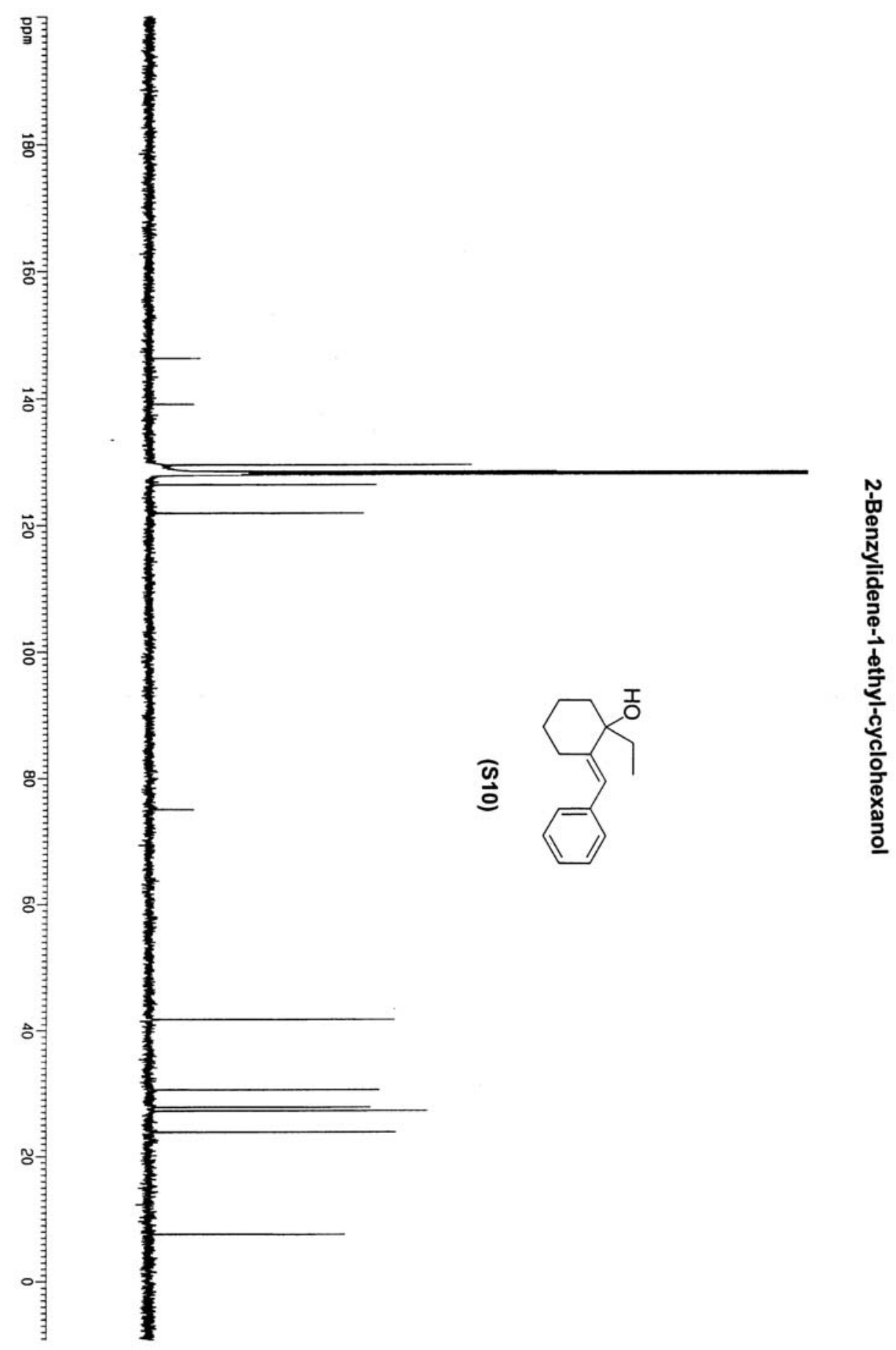




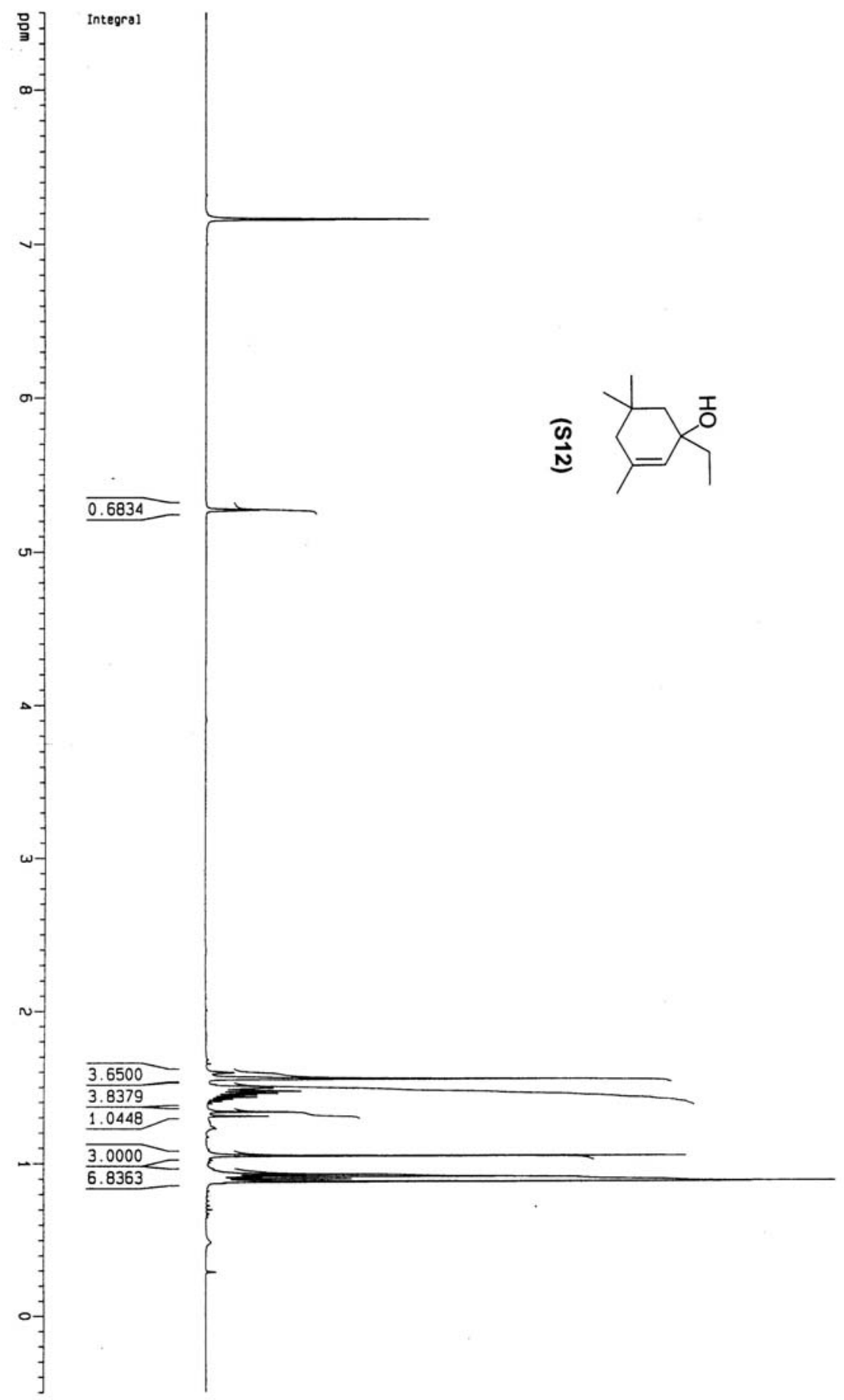

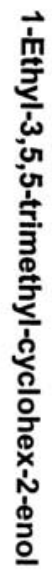




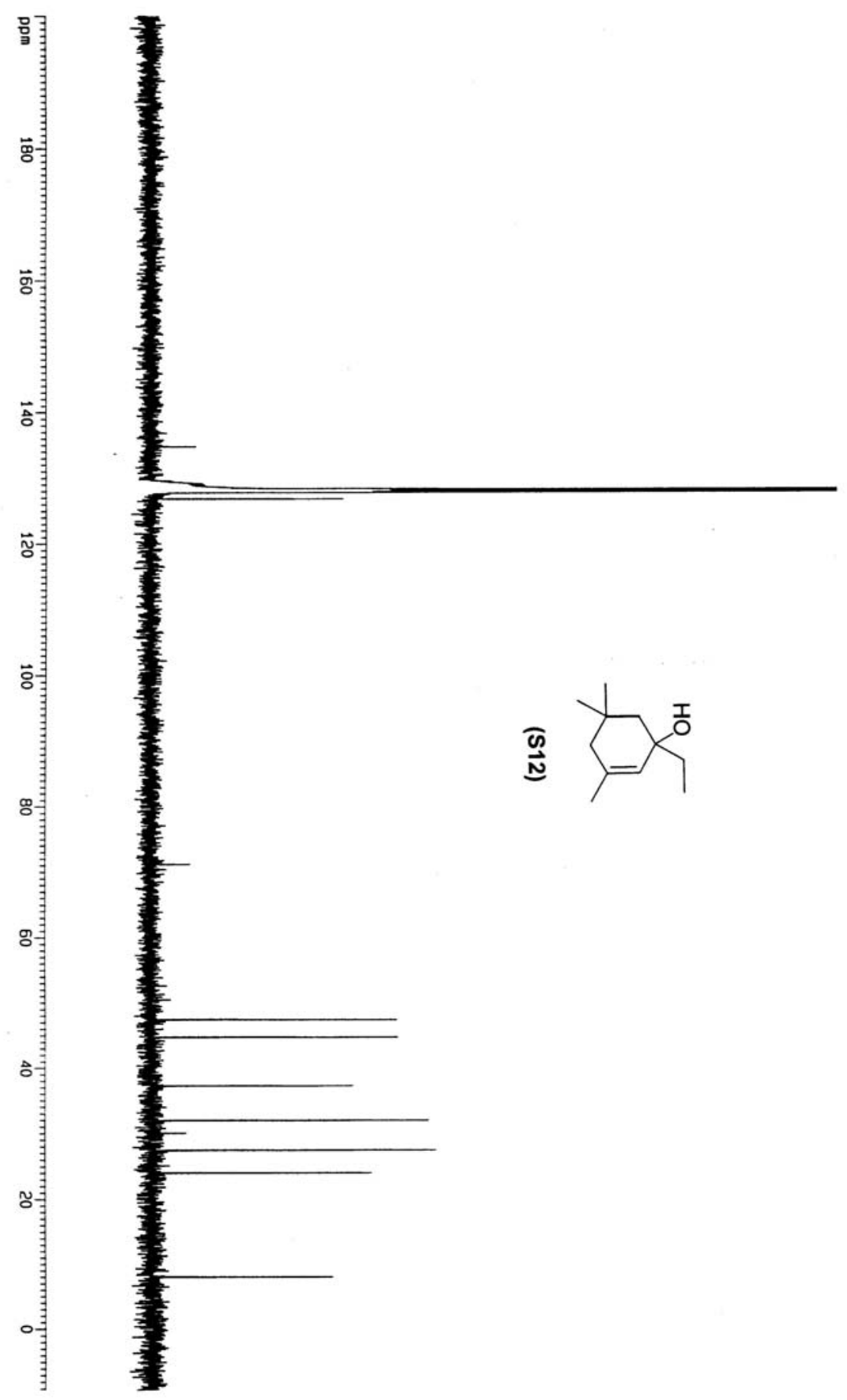

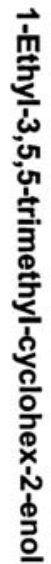




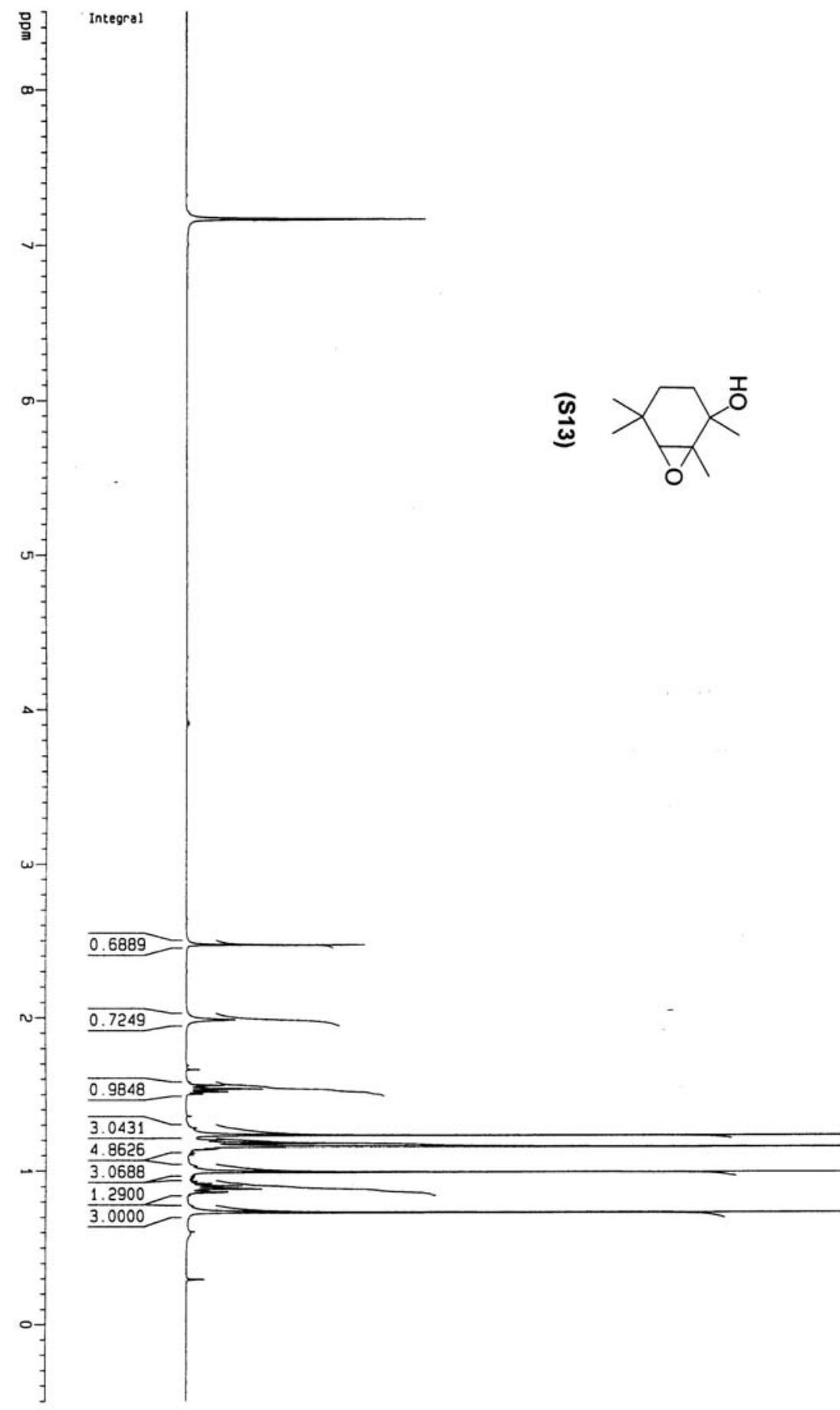

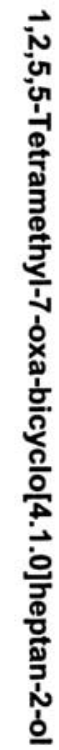




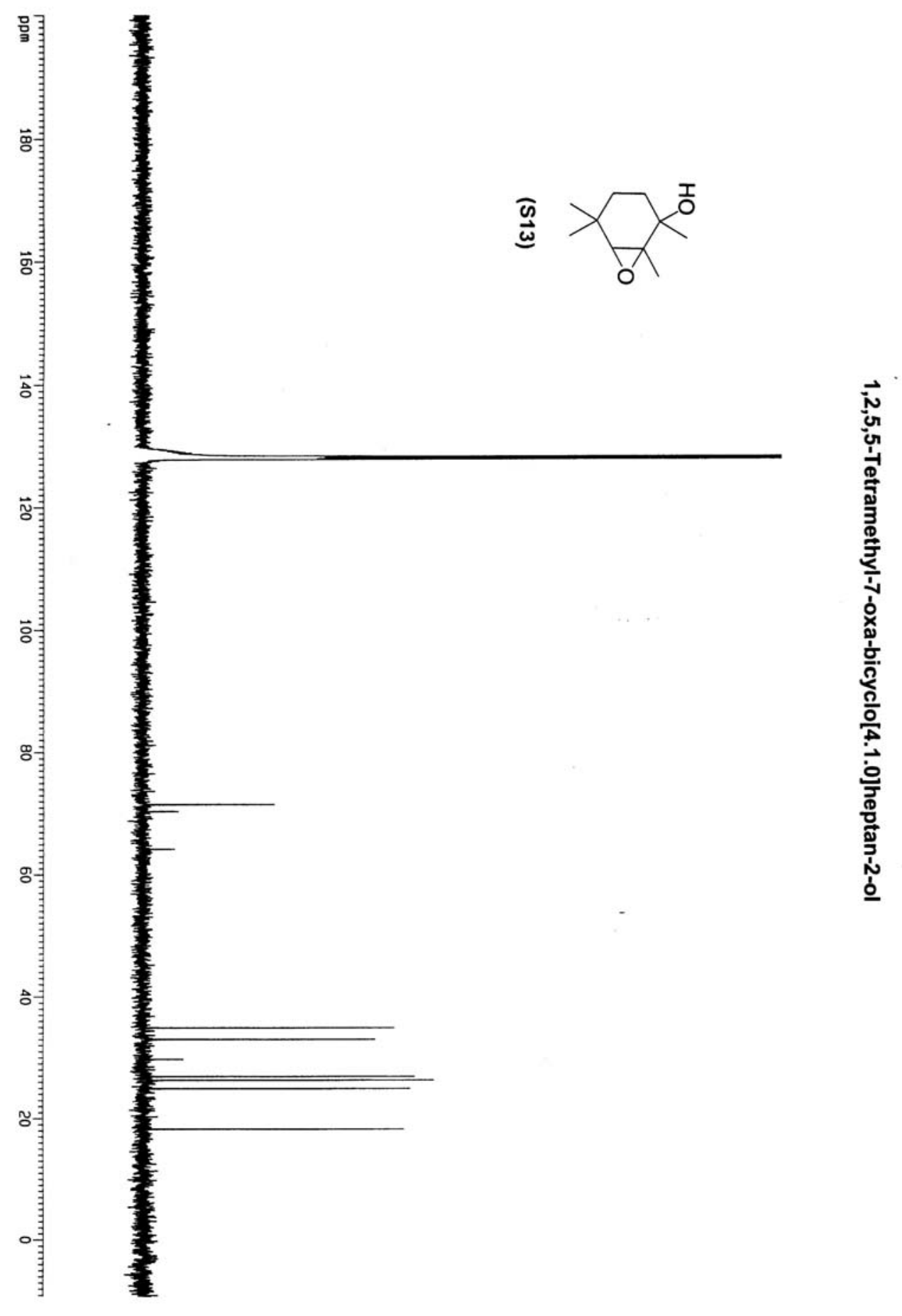



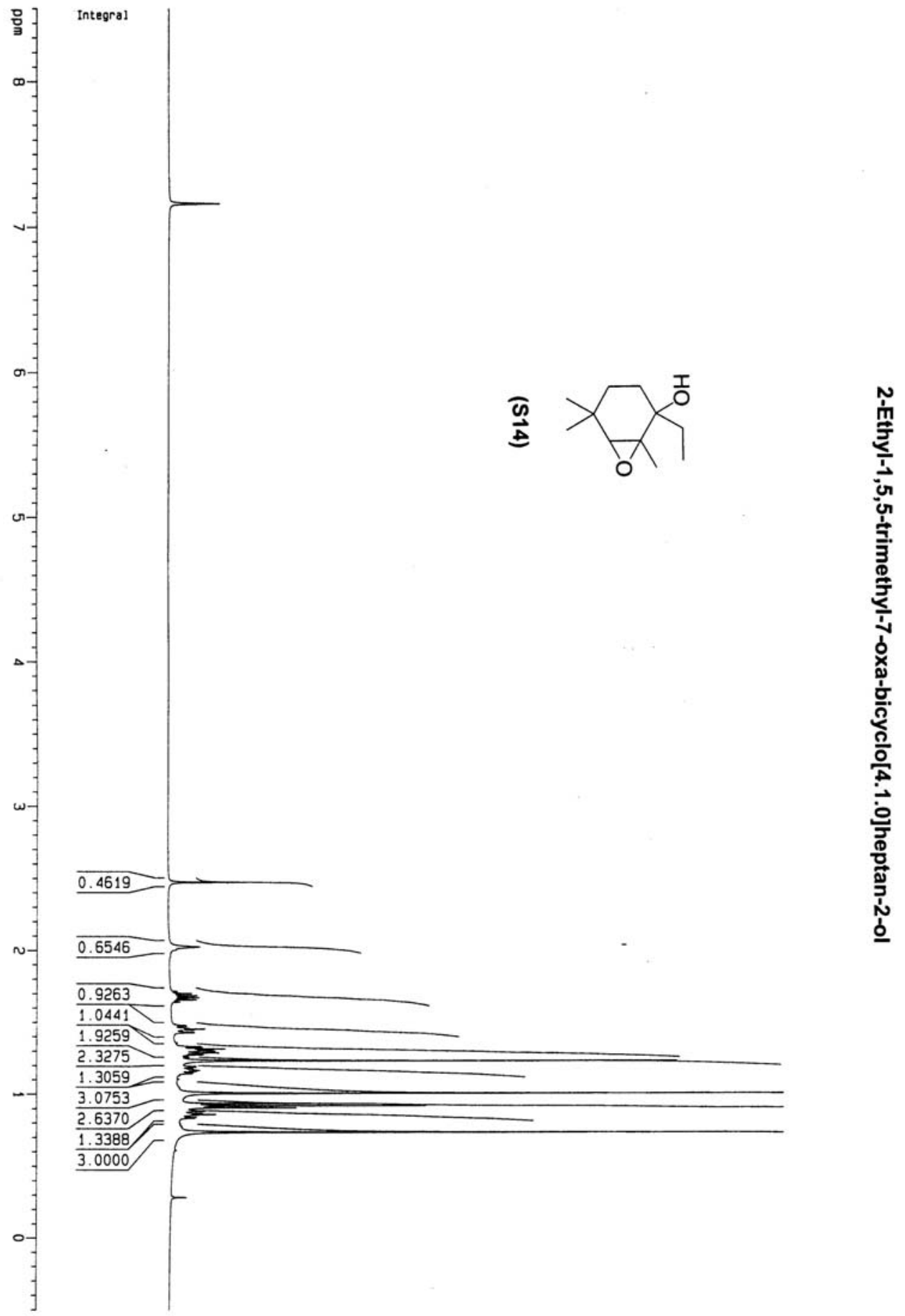


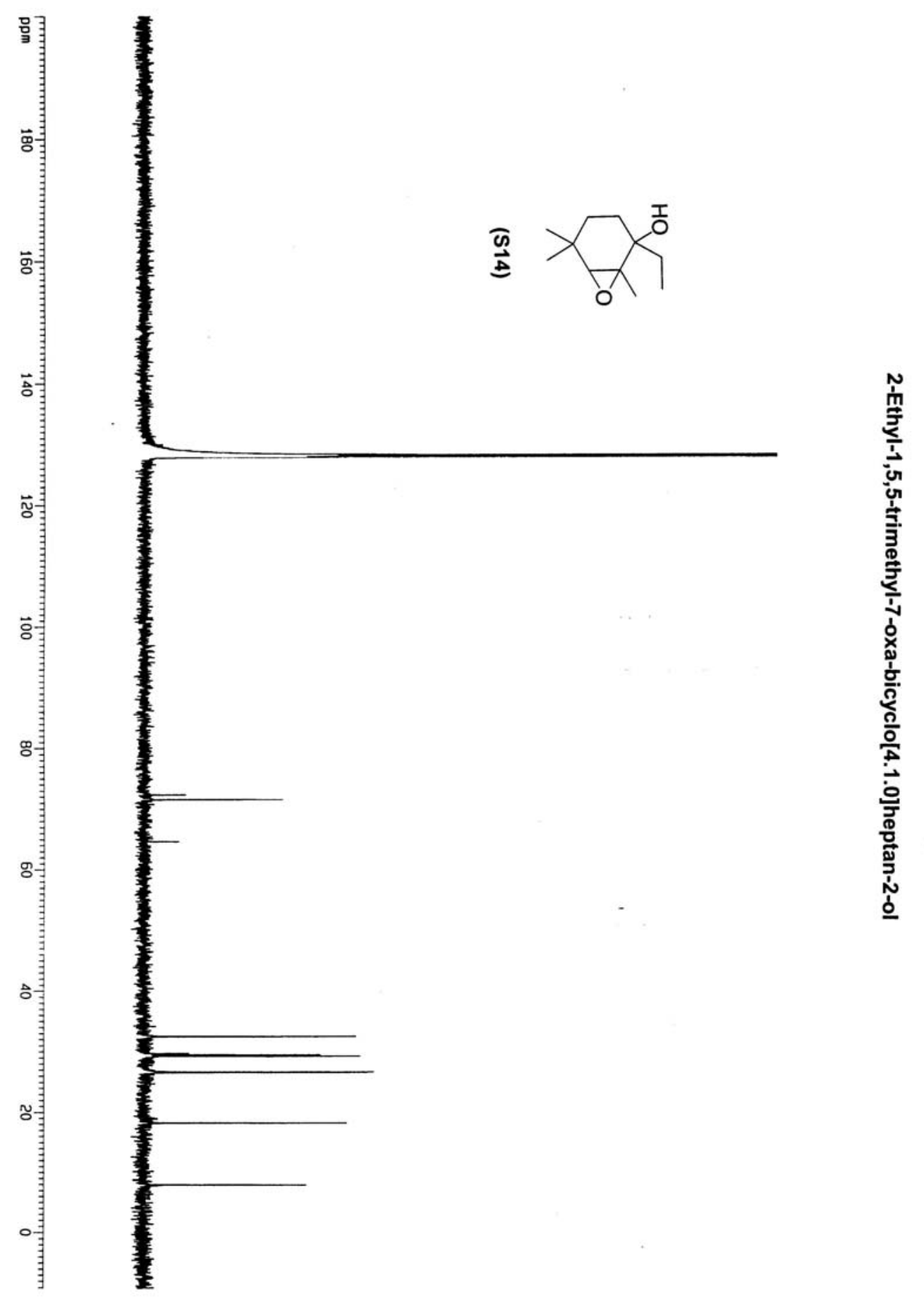




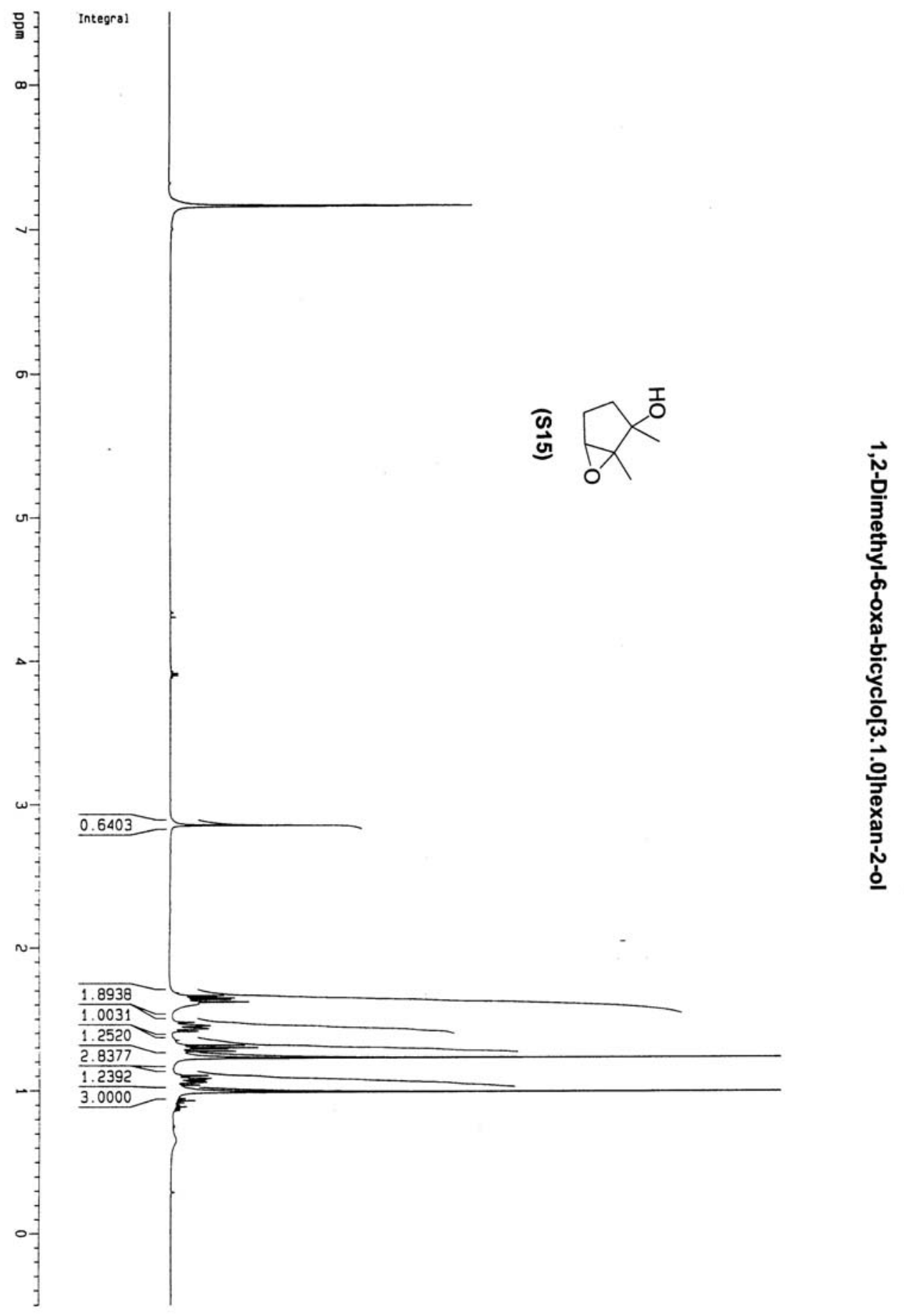




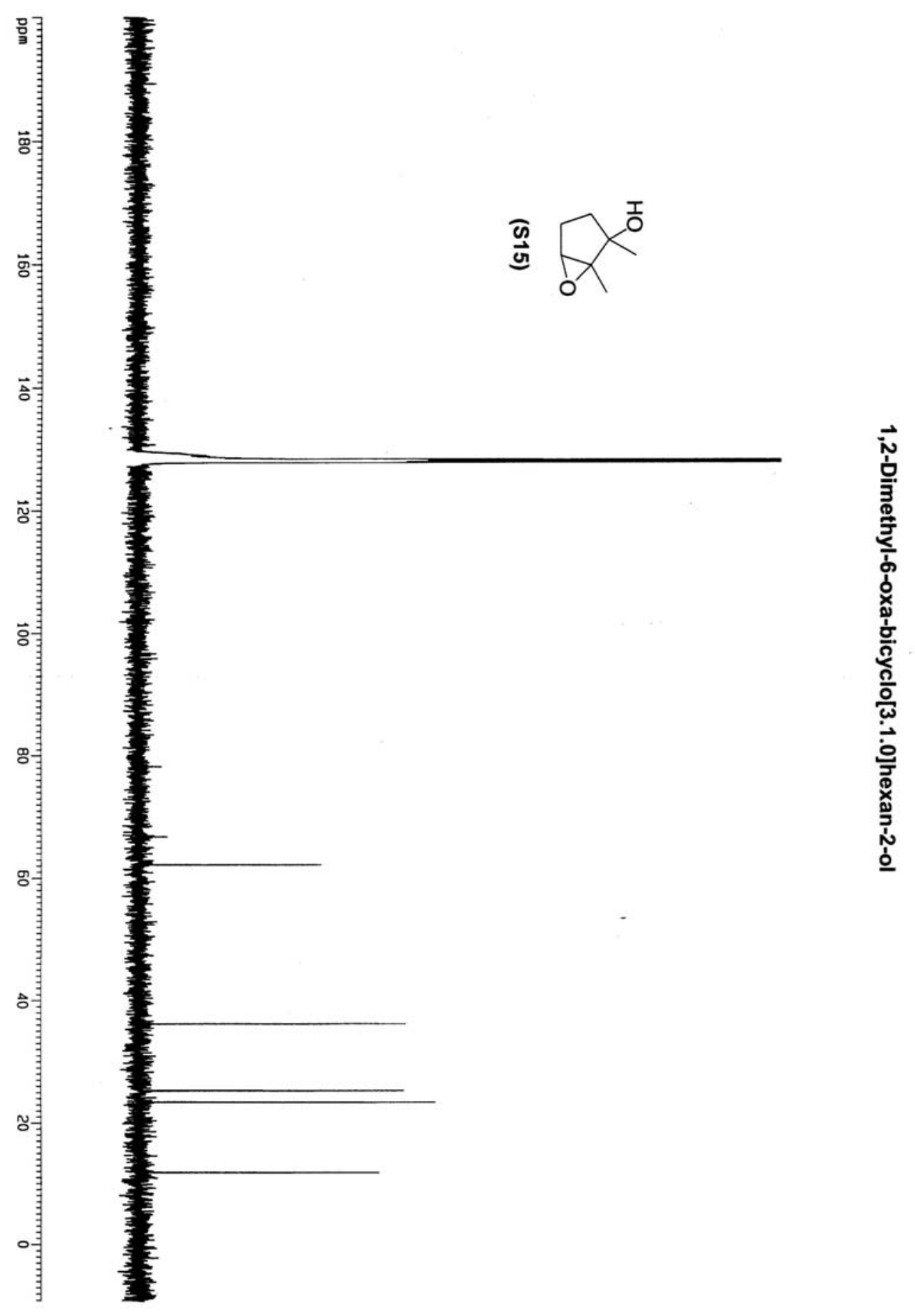




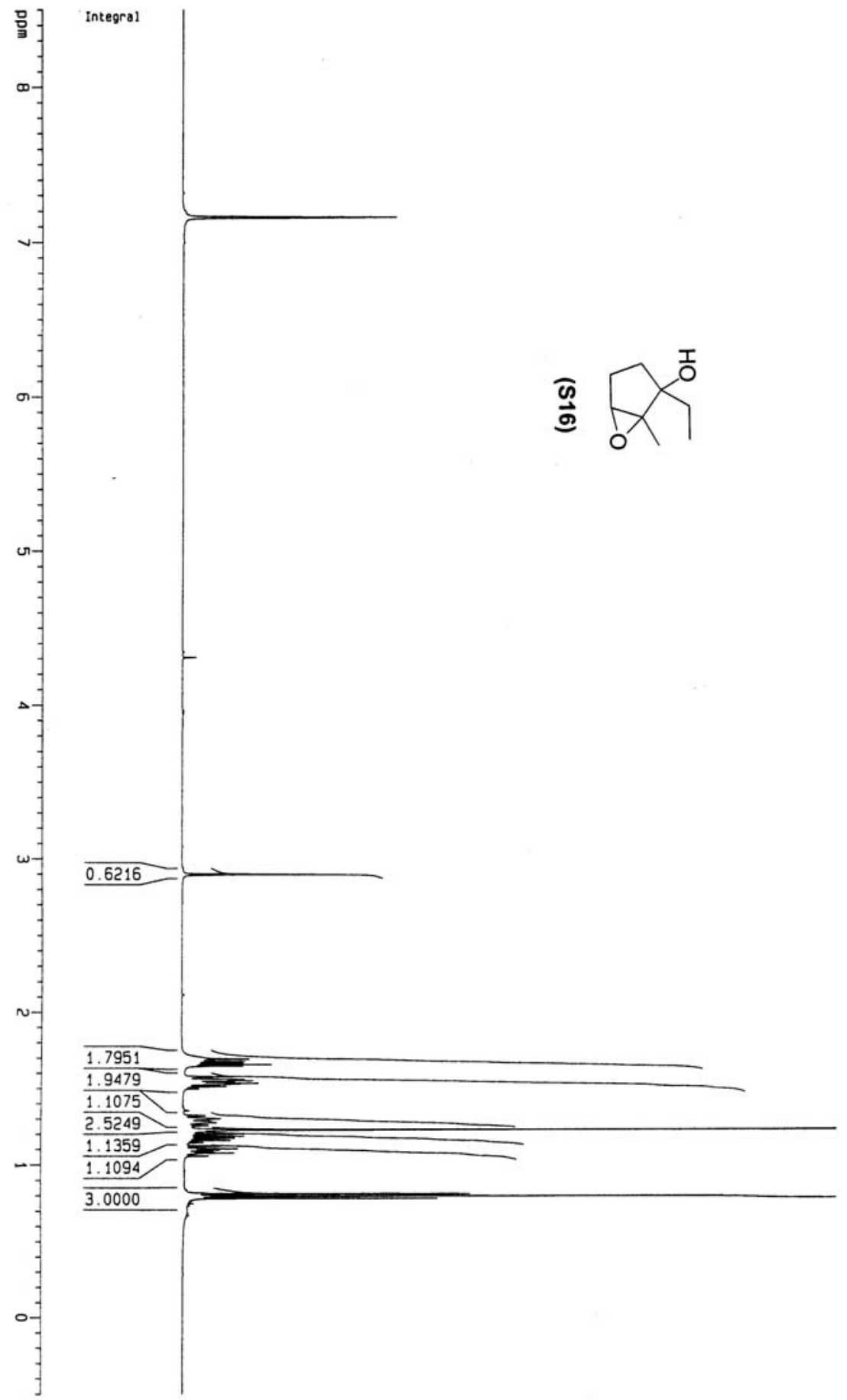

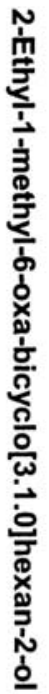




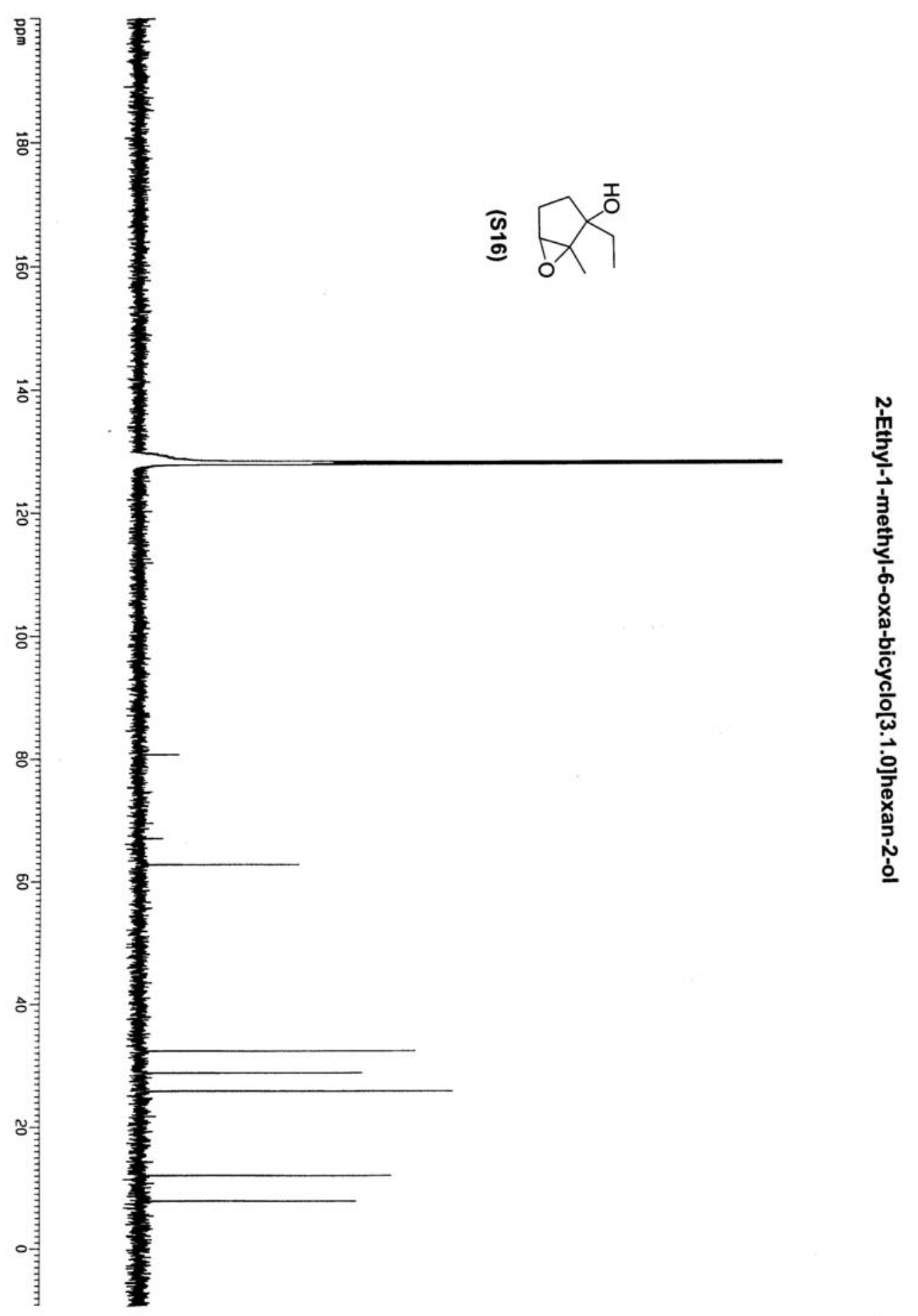



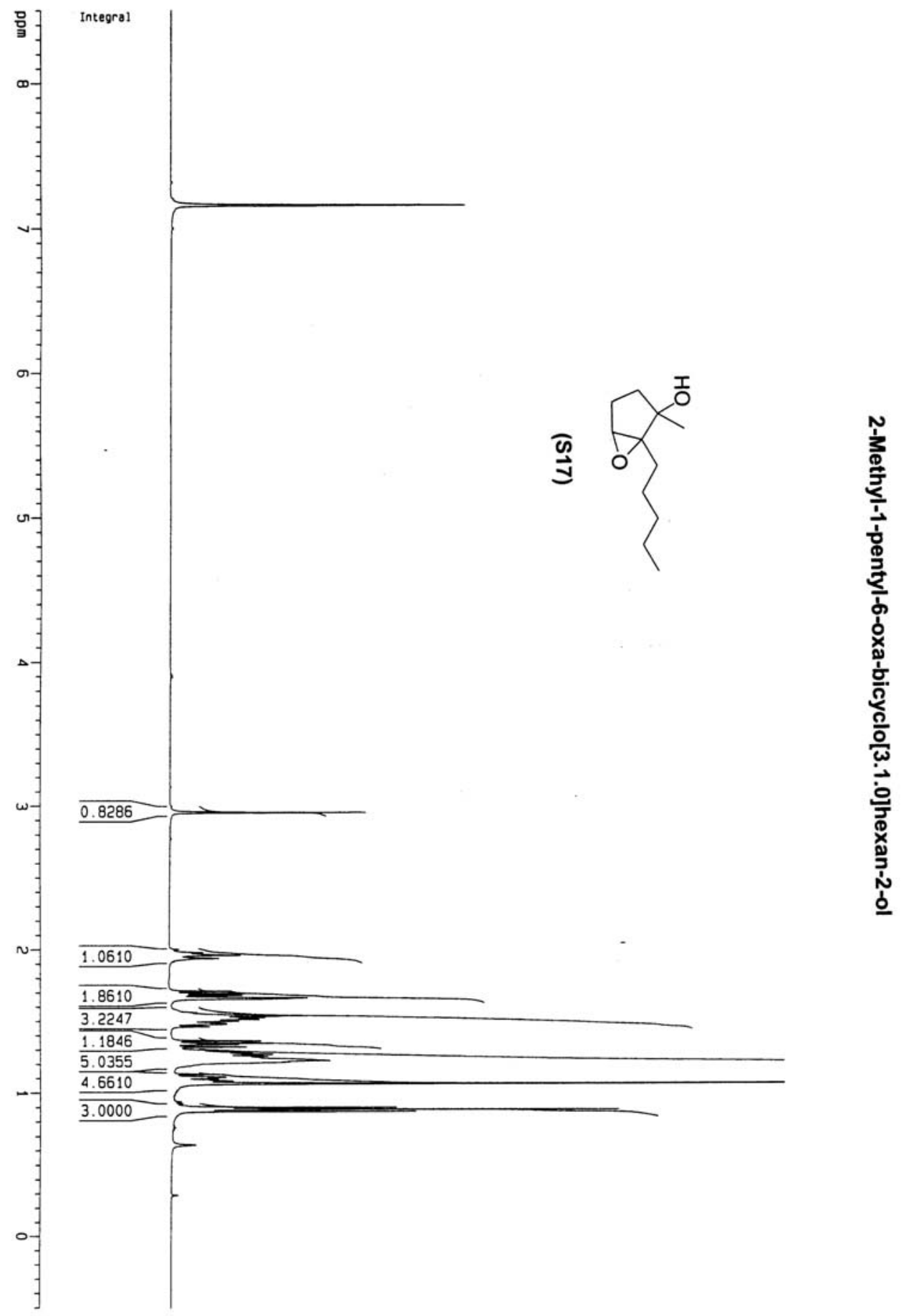


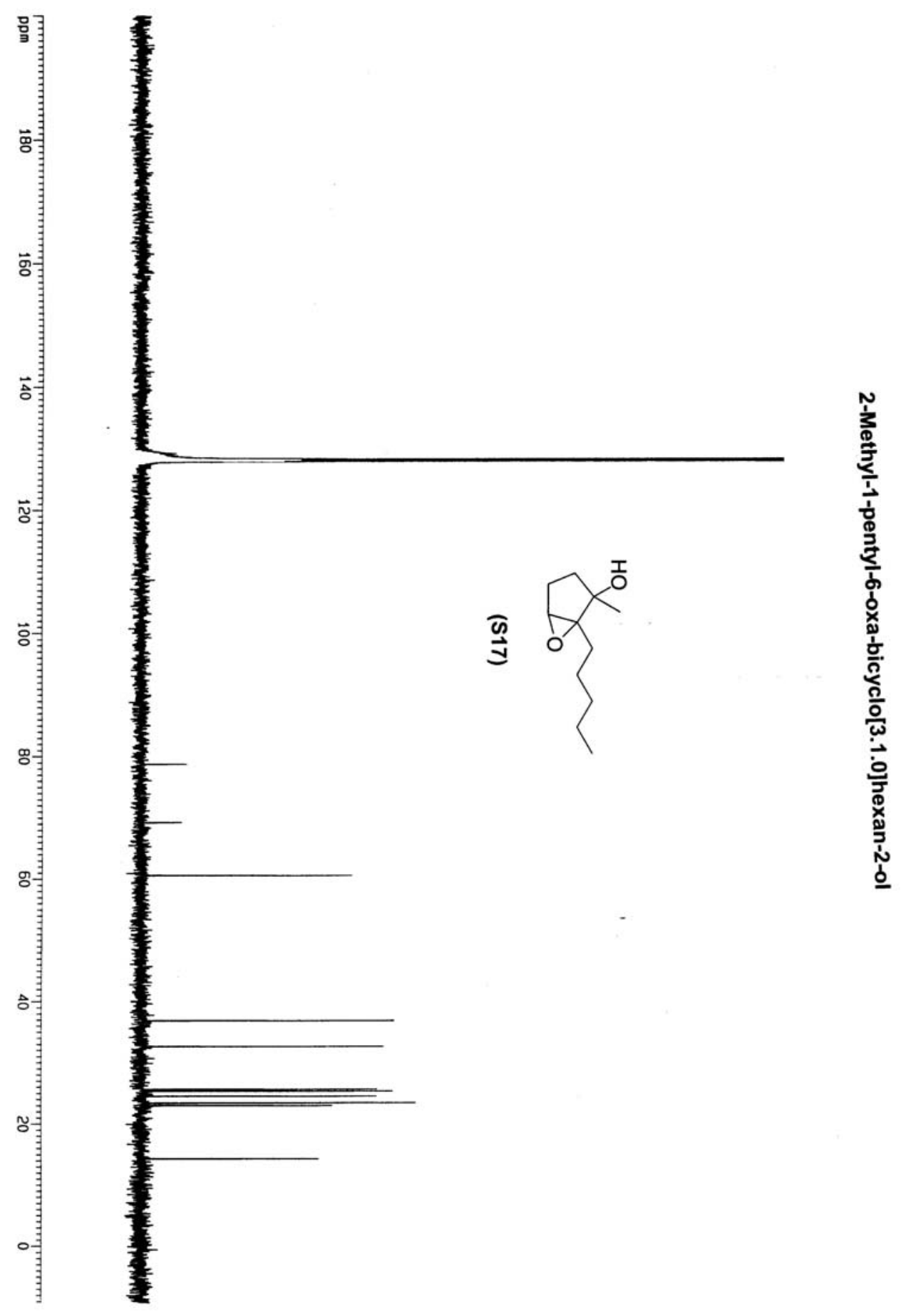




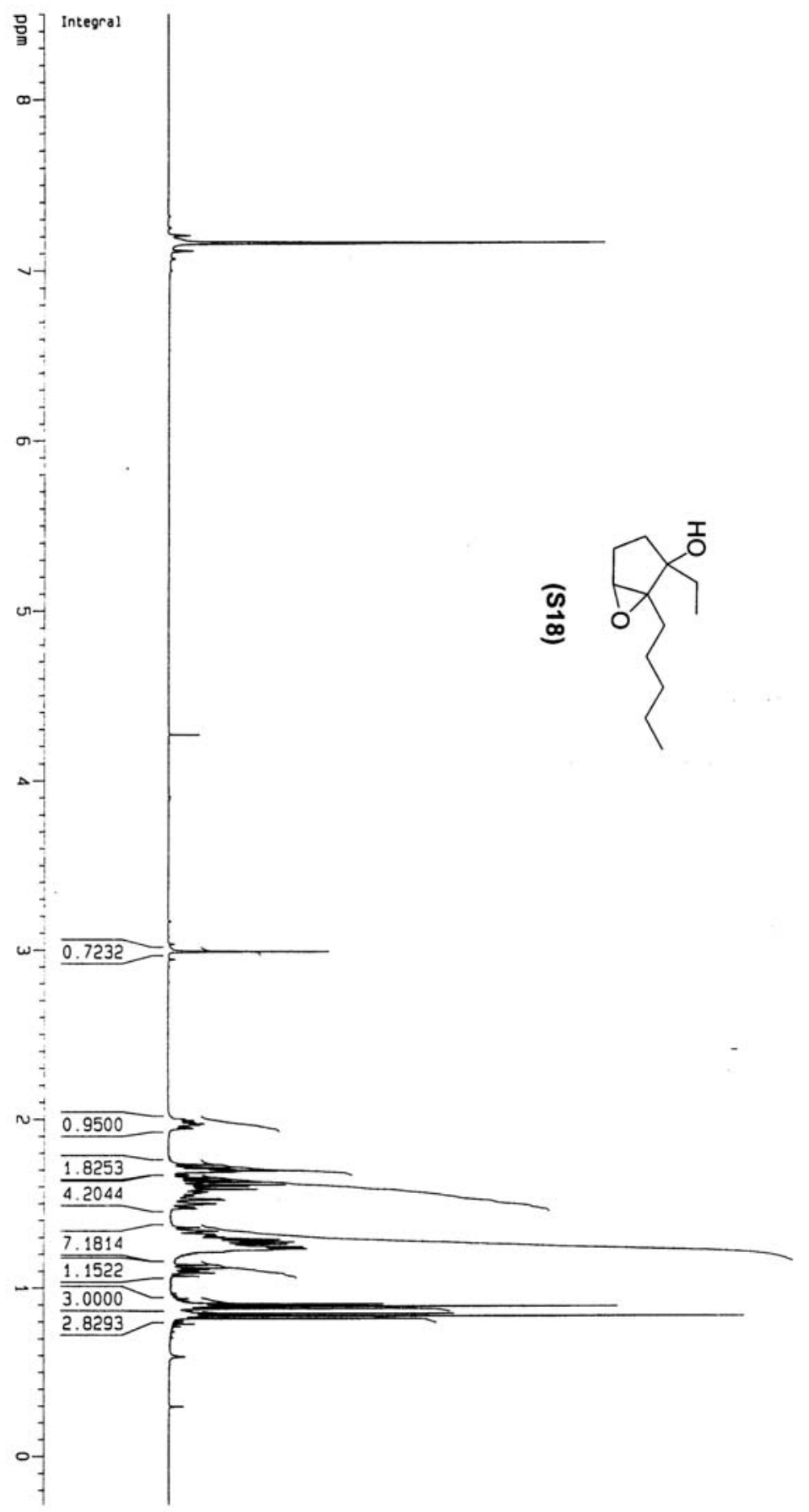

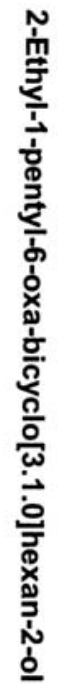




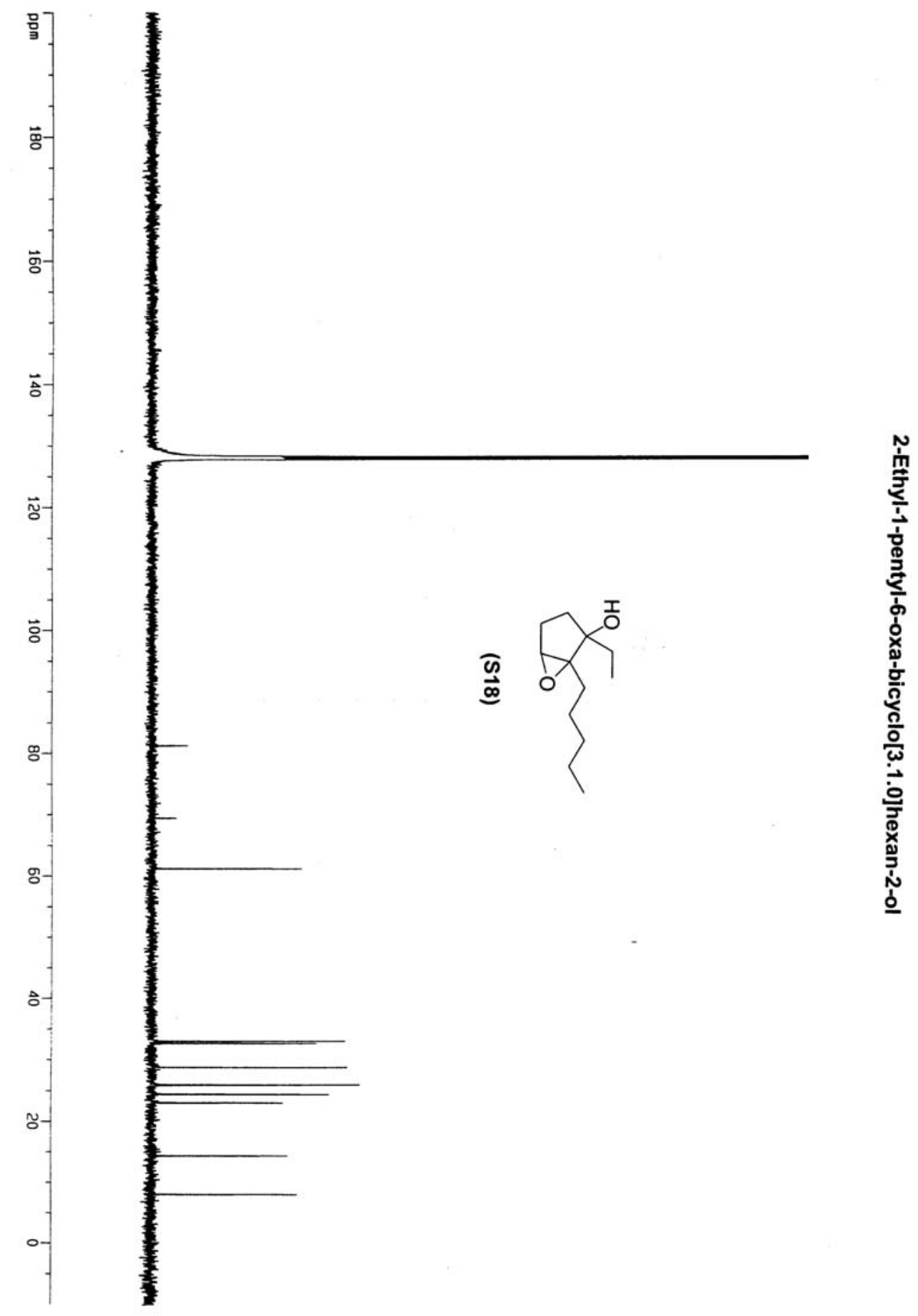




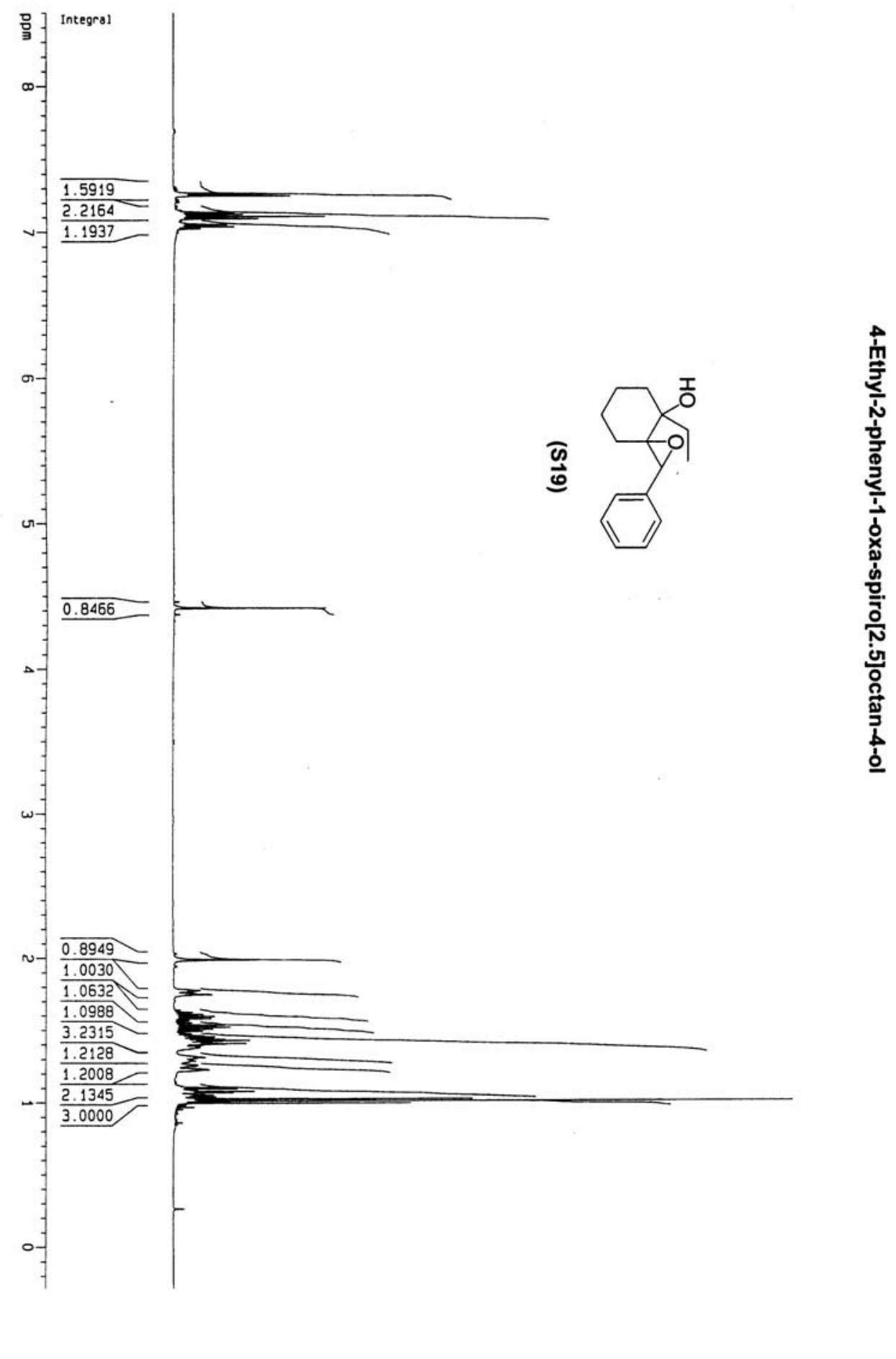




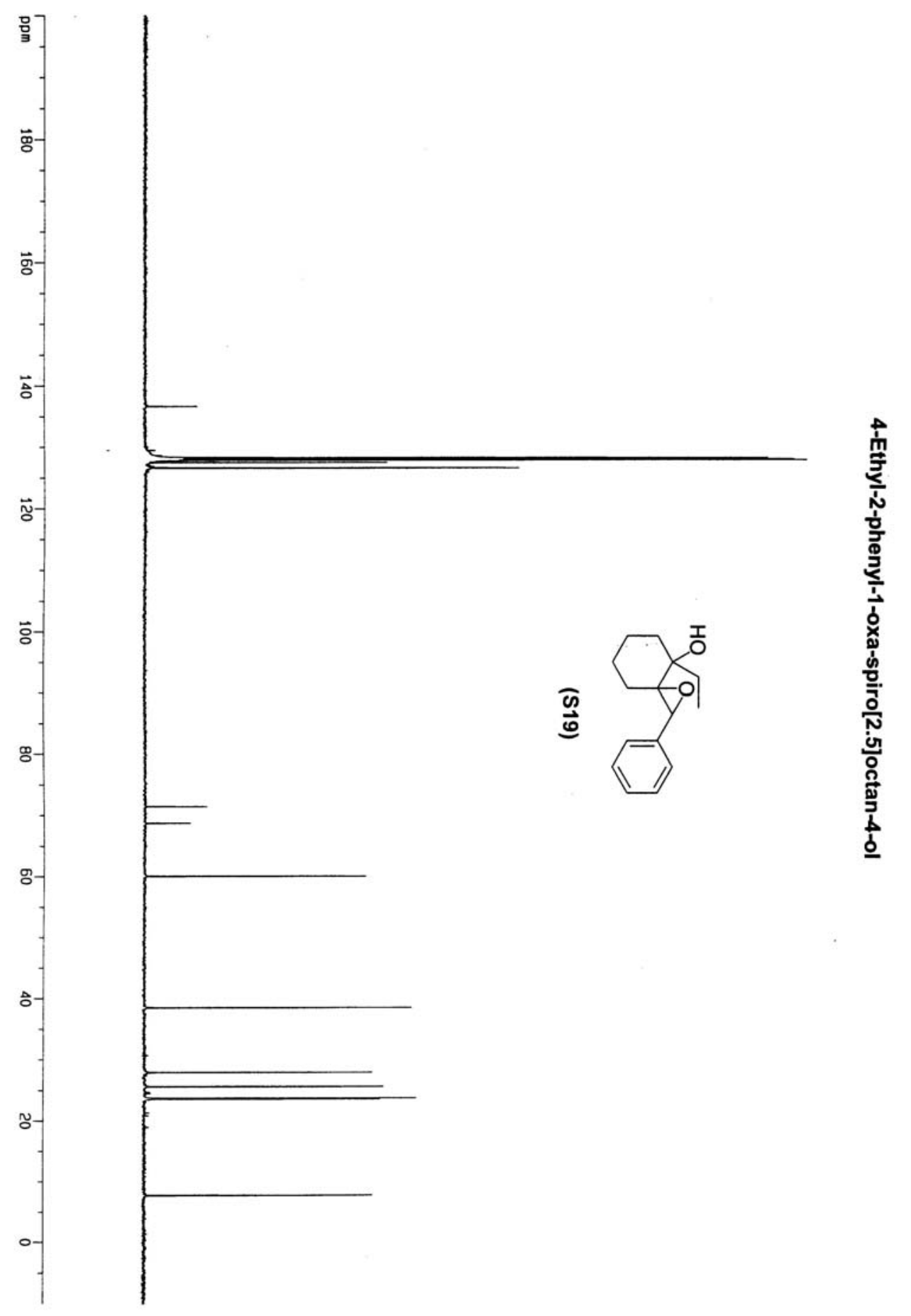




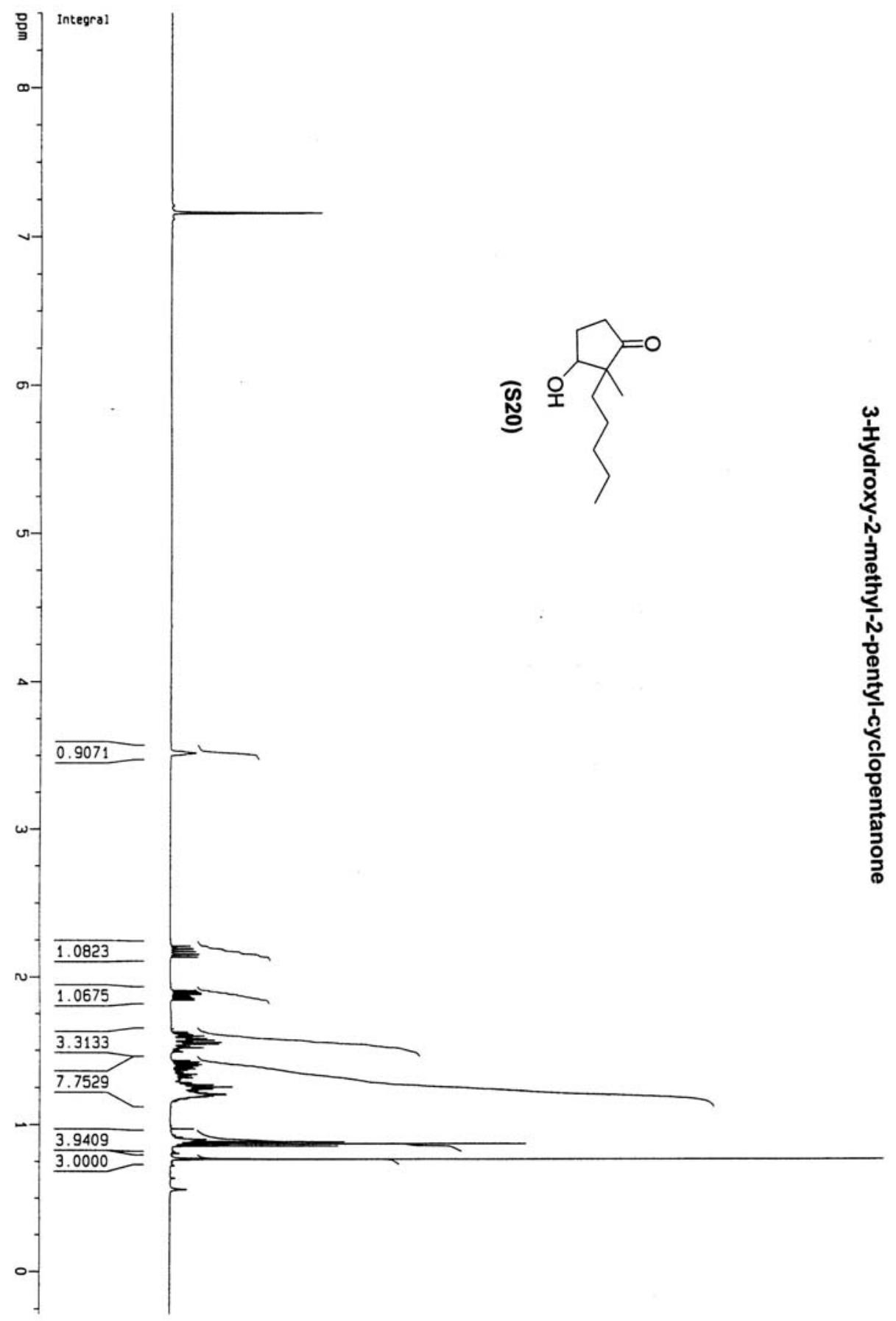




$$
\text { Li }
$$



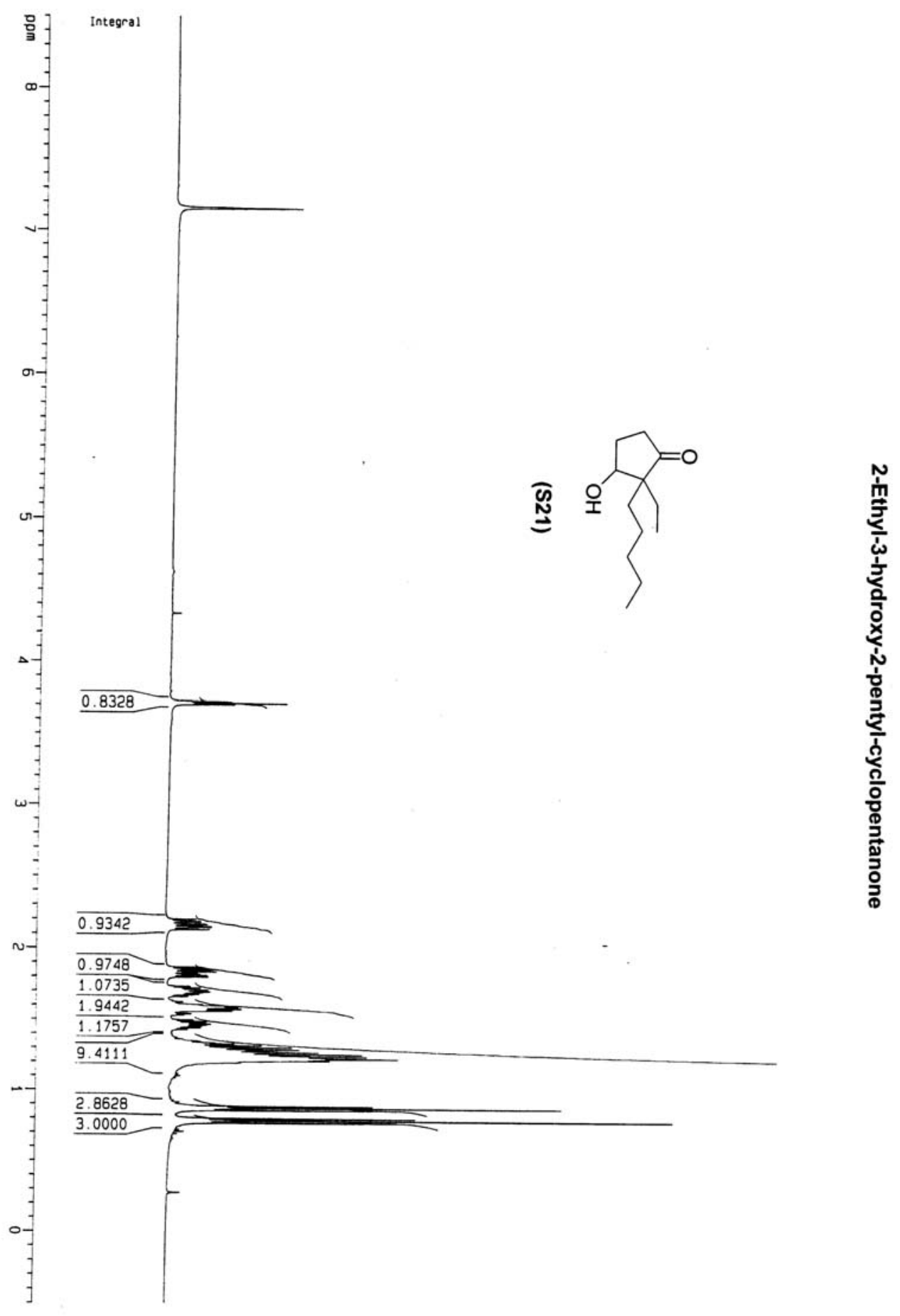


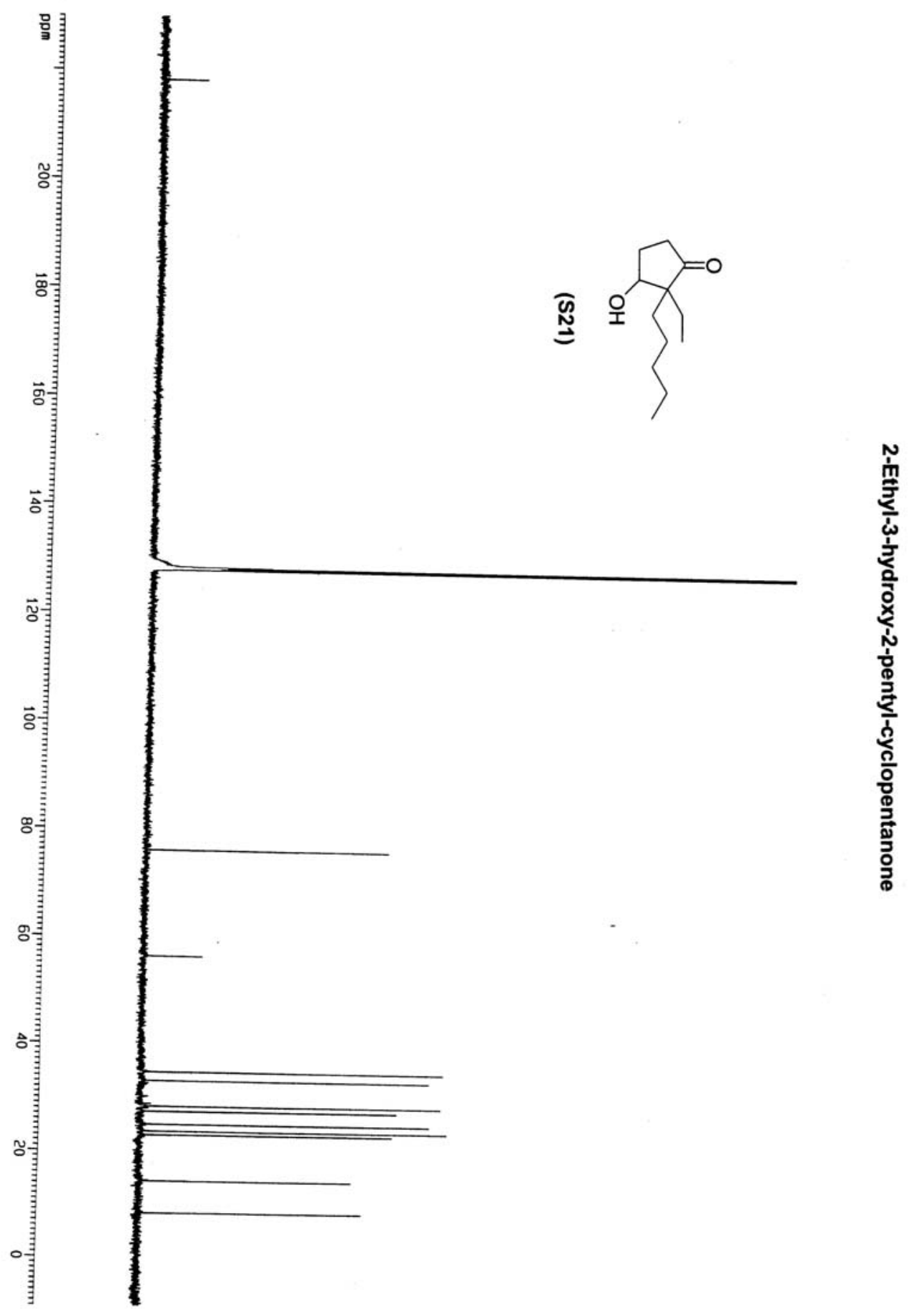




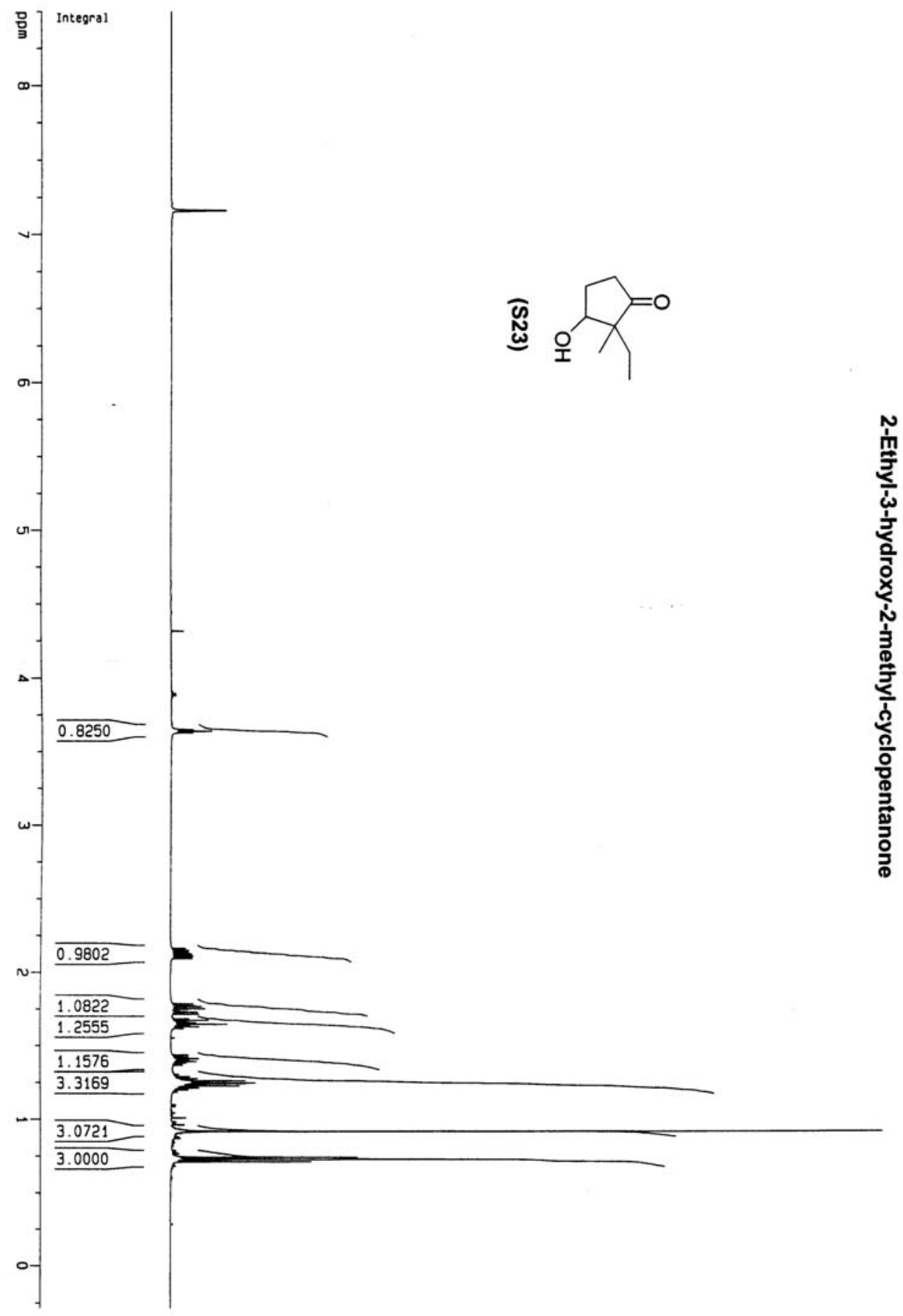




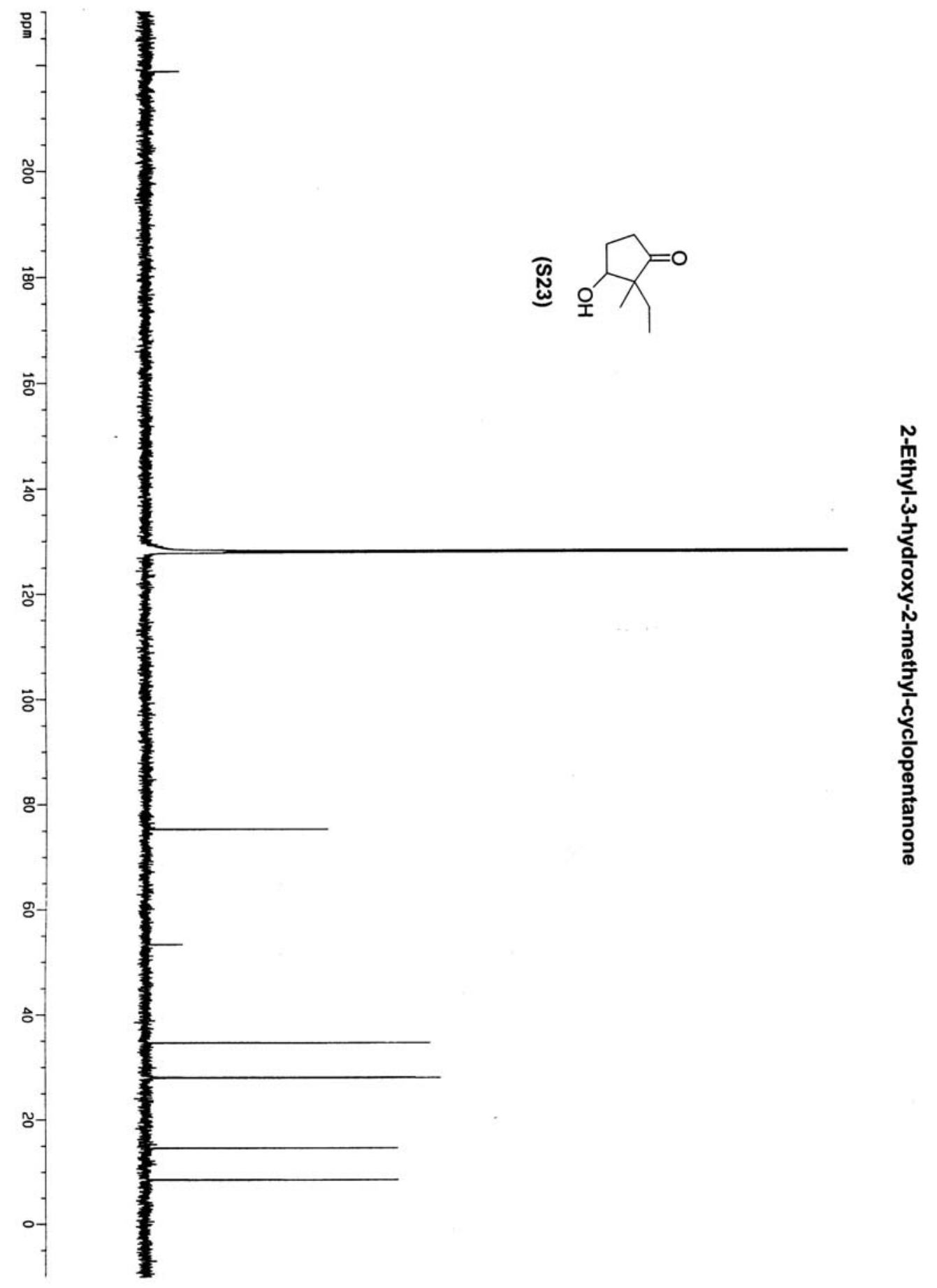

